Copyright (C) 2005 IEEE. Reprinted from

IEEE Transactions on Power Systems, 2005; 4 (20): 1886-1904

This material is posted here with permission of the IEEE. Such permission of the IEEE does not in any way imply IEEE endorsement of any of the University of Adelaide's products or services. Internal or personal use of this material is permitted. However, permission to reprint/republish this material for advertising or promotional purposes or for creating new collective works for resale or redistribution must be obtained from the IEEE by writing to pubs-permissions@ieee.org.

By choosing to view this document, you agree to all provisions of the copyright laws protecting it. 


\title{
Inclusion of Higher Order Terms for Small-Signal (Modal) Analysis: Committee Report-Task Force on Assessing the Need to Include Higher Order Terms for Small-Signal (Modal) Analysis
}

\author{
J. J. Sanchez-Gasca, Fellow, IEEE, V. Vittal, Fellow, IEEE, M. J. Gibbard, Life Fellow, IEEE, \\ A. R. Messina, Senior Member, IEEE, D. J. Vowles, Member, IEEE, S. Liu, Student Member, IEEE, and \\ U. D. Annakkage, Senior Member, IEEE
}

\begin{abstract}
This paper summarizes the work done by the Task Force on Assessing the Need to Include Higher Order Terms for Small-Signal (Modal) Analysis. This Task Force was created by the Power System Dynamic Performance Committee to investigate the need to include higher order terms for small signal (modal) analysis. The focus of the work reported here is on establishing and documenting the practical significance of these terms in stability analysis using the method of Normal Forms. Special emphasis was placed on determining and describing conditions when higher order terms need to be included to accurately describe modal interactions. Test cases were developed on a standard test system to demonstrate the application of appropriate indices to detect the occurrence of nonlinear interaction and hence the need for higher order terms in stability analyzes. The use of the higher order terms in the site selection for a damping controller is also documented.
\end{abstract}

Index Terms-Method of normal forms, modal analysis, modal damping, modal frequency, nonlinear modal interaction.

\section{INTRODUCTION}

$\mathbf{T}$ HIS paper, which serves as a committee report, describes the investigations conducted by the Task Force on Assessing the Need to Include Higher Order Terms for SmallSignal (Modal) Analysis established by the Power System Dynamic Performance Committee.

An important problem for the power industry is the presence of low-frequency electromechanical oscillations that often arise between interconnected areas in a power system. These oscillations are often poorly damped and have a negative impact on the power transfer capabilities of the system [1].

Manuscript received May 4, 2005; revised May 3, 2005. Paper no. TPWRD00259-2005.

J. J. Sanchez-Gasca is with GE Energy, Schenectady, NY 12345 USA (e-mail: juan.sanchez@ps.ge.com).

V. Vittal is with the Department of Electrical Engineering, Arizona State University, Tempe, AZ 85287 USA.

M. J. Gibbard and D. J. Vowles are with the School of Electrical and Electronic Engineering, The University of Adelaide, Adelaide 5005, Australia.

A. R. Messina is with the Center for Research and Advanced Studies, National Polytechnic Institute, Guadalajara 45090, Mexico.

S. Liu is with the Department of Electrical and Computer Engineering, Iowa State University, Ames, IA 50011 USA.

U. D. Annakkage is with the Department of Electrical and Computer Engineering, University of Manitoba, Winnipeg, MB R3T 5V6, Canada.

Digital Object Identifier 10.1109/TPWRS.2005.858029
Small-signal stability analysis is the conventional analysis tool for studying electromechanical oscillations. This type of analysis provides an understanding of the underlying modal structure of a power system and gives insights into a system's dynamic characteristics that are not easily obtained from time domain simulations. However, small-signal stability analysis is based on a first-order (linear) approximation of the nonlinear power system differential-algebraic equations in the neighborhood of an operating point. Such an approximation has two important consequences: on the one hand, it allows for the application of powerful linear analysis methods well suited for the study of large systems. On the other hand, it limits the scope of the analysis to the region where the linear approximation is valid.

It is well known that the manner in which the state variables and the modes in a power system interact is a complex phenomenon. It has been suggested that in certain cases, such as when the system is stressed, linear analysis techniques might not provide an accurate picture of the power system modal characteristics [2]. This makes techniques that extend the domain of applicability of small-signal stability analysis an attractive proposition for advancing the understanding of power system dynamics. Of particular interest is the study of modes and interactions that result from the combination of the individual system modes of the linearized system. These modes and their interactions are termed "higher order modes" and "higher order modal interactions," respectively.

In the period 1996 to 2001, investigators from Iowa State University published numerous papers [3]-[13] and made presentations at various PES forums advocating the application of Normal Forms as a means for studying higher order modal interactions. Recognizing the potential value of such techniques, the Task Force on Assessing the Need to Include Higher Order Terms for Small-Signal (Modal) Analysis was formed by the Power System Stability Subcommittee of the Power System Dynamic Performance Committee.

The Task Force was given the assignment of investigating the practical significance of higher order modal interactions. More specifically, the ensuing work was aimed at providing answers to the following questions. 
1) What is the practical significance of higher order modal interactions?

2) When should second-order or higher modal analysis be used?

3) What are the engineering insights, not obtainable from linear analysis, that second-order analysis provides?

4) When should linear analysis be complemented with a higher order approximation?

5) What are the computational requirements for higher order modal analysis of realistic system models?

6) What is the range of applicability of second-order analysis?

The work performed by the Task Force was focused on the application of the method of Normal Forms to the analysis of higher order modal interactions in the four generator system introduced in [14]. Developing fully worked examples, using an accepted test system, provided concrete means for enhancing and confirming the understanding of the techniques as currently applied. The emphasis of this effort was placed on gaining a better understanding of the insights provided by the method and on developing guidelines for analyzing higher order modes in the frequency range associated with local and inter-area modes.

Results obtained for two selected cases are used to highlight the applicability of the method of Normal Forms to the analysis of nonlinear modal interactions. The difference between the two cases is the fault duration. In the first case the fault duration is 10 $\mathrm{ms}$ and, in the second case, $19 \mathrm{~ms}$. This allows for the study of the effect that the system stress has on the modal characteristics, and to determine whether the increased stress, due to a longer fault duration, results in nonlinear modal interactions that can be predicted by the Normal Forms analysis. The application of various indices used in conjunction with the Normal Forms analysis to predict and evaluate the onset of nonlinear modal interactions is documented. The modal interactions predicted by the method of Normal Forms were verified by a frequency domain analysis of the time domain responses.

A concrete example of a situation that specifically addresses question 3 above is provided in Appendix B based on a heavily stressed system. The case is studied using linear (conventional) and nonlinear modal analyzes techniques to determine suitable locations for controllers to damp the inter area mode of oscillation. Normal Forms analysis shows that nonlinear modal interaction plays an important role and that the location suggested by the nonlinear measures obtained from the Normal Forms analysis provides more effective damping of the inter area mode.

The paper is organized as follows: Section II outlines the formulation of the method of Normal Forms used for power system analysis, and describes several indices and performance measures. The test system is briefly described in Section III. Section IV is the main section and presents the Normal Form analysis on two different test cases. A discussion on practical aspects associated with the Normal Forms analysis is provided. The conclusions of the work are presented in Section V. An important complement to the main body of the paper are Appendices $\mathrm{A}$ and $\mathrm{B}$. The former highlights differences and similarities between linear and nonlinear analyzes. Appendix B presents recent developments pertaining to the siting of controllers on a highly stressed system using nonlinear measures with the objective of damping electromechanical oscillations.

\section{METHOD OF NORMAL FormS}

The method of Normal Forms was introduced by Poincaré and is a well established mathematical procedure for simplifying nonlinear differential equations. Using this method, provided that certain conditions are met [15], a set of nonlinear differential equations can be transformed, up to a specified order, into a set of linear differential equations by performing a sequence of nonlinear coordinate transformations. The transformed equations are in their simplest form, i.e., in their Normal Form, and allow for the study of essential modal characteristics. An important characteristic of this approach is that it provides a closed form solution for the system state variables. These last two features are key elements in the analysis of power system stability.

A brief overview of the method of Normal Forms is provided first. The purpose of the overview is to highlight aspects relevant to power system modal analysis. For a more formal introduction to the subject, the reader is referred to [15]-[19]. Following the description of the Normal Forms method, several indices for quantifying higher order modal characteristics are introduced.

In a manner similar to small-signal stability analysis, the method of Normal Forms begins by performing a Taylor series expansion of the nonlinear system of interest, $\dot{x}=f(x)$, in the neighborhood of a stable operating point (This expansion can also be done in the neighborhood of an unstable equilibrium point [6]). However, unlike small-signal stability analysis the expansion is carried out beyond the first-order (linear) term. Although there is no theoretical limit to the number of terms in the series, the computation of higher order terms for a typical power system represents a heavy computational burden. For this reason, the Taylor series expansion is usually computed up to second-order terms

$$
\dot{x}=A x+X_{2} .
$$

The state-space equation for the $i$ th state variable is given by

$$
\dot{x}_{i}=A_{i} x+\frac{1}{2} x^{T} H^{i} x .
$$

In (2), $A_{i}$ is the $i$ th row of the Jacobian matrix $[\partial f / \partial x]$, and $H^{i}$ is a Hessian matrix. The $j k$ th element of $H^{i}$ is given by

$$
H_{j k}^{i}=\frac{\partial^{2} f_{i}}{\partial x_{j} \partial x_{k}} .
$$

The next step is to transform (1) to its Jordan form by applying the similarity transformation $x=U y$, where $U$ is the matrix of right eigenvectors of $A$. This transformation yields

$$
\dot{y}=\Lambda y+\frac{1}{2} V^{T}\left[\begin{array}{c}
y^{T} U^{T} H^{1} U y \\
y^{T} U^{T} H^{2} U y \\
\ldots \\
y^{T} U^{T} H^{n} U y
\end{array}\right] .
$$

The elements of the diagonal matrix $\Lambda$ are the eigenvalues of $A, \lambda_{1}, \lambda_{2}, \ldots, \lambda_{n}$, and $V$ is the matrix of left eigenvectors of 
$A$. The state equation for the $j$ th Jordan form variable is of the form

$$
\dot{y}_{j}=\lambda_{j} y_{j}+\sum_{k=1}^{n} \sum_{l=1}^{n} C_{k l}^{j} y_{k} y_{l} .
$$

$C_{k l}^{j}$ is the $k l$ th element of the matrix $C^{j}$

$$
C^{j}=\frac{1}{2} \sum_{p=1}^{n} v_{j p}\left[U^{T} H^{p} U\right] .
$$

The term in the brackets is an $n \times n$ matrix; $v_{j p}$ is the $j p$ th element of the matrix $V$. Notice that the terms of the summation in (5) constitute a homogeneous polynomial of second order, i.e., all the terms of the polynomial are of second order.

The next step is to transform (5) into a simpler form, its Normal Form, by eliminating the nonlinear terms. The transformation is of the form [15]

$$
y=z+h 2(z)
$$

where $z$ is the vector of Normal Form state variables. The expanded form of (7) is given by

$$
y_{j}=z_{j}+\sum_{k=1}^{n} \sum_{l=1}^{n} h 2_{k l}^{j} z_{k} z_{l} .
$$

$h 2_{k l}^{j}$ is given by

$$
h 2_{k l}^{j}=\frac{C_{k l}^{j}}{\lambda_{k}+\lambda_{l}-\lambda_{j}} \quad j, k, l=1,2, \ldots, n
$$

provided that $\lambda_{k}+\lambda_{l}-\lambda_{j} \neq 0$. The transformation (7) removes the second-order terms from (4). This is referred to as the nonresonant condition. Notice that $h 2(z)$ contains only secondorder polynomial terms in $z$.

The transformed equation is a set of decoupled first-order linear differential equations

$$
\dot{z}=\Lambda z+o(3) ; \quad \dot{z}_{j}=\lambda_{j} z_{j}+o(3), \quad j=1,2, \ldots, n
$$

where $o(3)$ denotes third- and higher order terms, and it is assumed that the second-order terms have been annihilated by the nonlinear transformation (7). By neglecting these terms, the linear solutions of the individual Normal Form variables $z_{j}(t)$ is given by

$$
z_{j}(t)=z_{j 0} e^{\lambda_{j} t} .
$$

In (11), $z_{j 0}$ is the initial condition of $z_{j}$. The initial conditions for $z$ are computed by solving the nonlinear equation (7), for a given initial condition $y_{0}$. The solution is obtained by formulating a nonlinear system of equations of the form

$$
f(z)=z-y_{0}+h 2(z)=0 .
$$

The solution $z$ to the above equation provides the initial condition $z_{0}$. This system of equations is directly related to the nature of the second-order nonlinear terms and describes how the $z$-variables differ from $y_{0}$ as a result of the nature of the $h 2$ terms. The numerical solution of these equations is complicated.
It requires a robust algorithm and is sensitive to the choice of the initial conditions for the solution. A robust solution technique has been developed and demonstrated for the test system chosen in Section IV-A. It is recommended that $y_{0}$ be selected as the choice of the initial condition for the algorithm described in Section IV A. This choice is based on the nature of the system of (12) where the variables $z$ differ from $y_{0}$ according to the second-order nonlinear terms. In Section IV, a detailed discussion of the initial condition solution is provided.

The solution to the original set of equations, (1), is obtained by transforming the $z$ variables back to the original state variables $x$. This is done by first using (7) to compute $y_{j}(t)=$ $z_{j 0} e^{\lambda_{j} t}+\sum_{k=1}^{n} \sum_{l=1}^{n} h 2_{k l}^{j} z_{k 0} z_{l 0} e^{\left(\lambda_{k}+\lambda_{l}\right) t}$, followed by the application of the similarity transformation $x=U y$ to compute $x$. This yields

$x_{i}(t)=\sum_{j=1}^{n} u_{i j} z_{j 0} e^{\lambda_{j} t}+\sum_{j=1}^{n} u_{i j}\left[\sum_{k=1}^{n} \sum_{l=1}^{n} h 2_{k l}^{j} z_{k 0} z_{l 0} e^{\left(\lambda_{k}+\lambda_{l}\right) t}\right]$.

In (13), $u_{i j}$ is an element of the matrix of right eigenvectors $U$.

Equation (13) constitutes the basis for most of the work performed to-date using the Normal Forms method for the study of power system electromechanical oscillations. The reason is evident: (13) clearly shows the relation between the state variables $x_{1}, \ldots, x_{n}$, the individual system modes $\lambda_{1}, \lambda_{2} \ldots \lambda_{n}$, and the second-order modes, $\lambda_{1}+\lambda_{1}, \lambda_{1}+\lambda_{2}, \ldots, \lambda_{n-1}+\lambda_{n}, \lambda_{n}+\lambda_{n}$.

The terms associated with the mode pairs $\lambda_{k}+\lambda_{l}$ provide information not available from the linear approximation of the power system equations. These terms represent "modal interactions" that arise due to the inclusion of the higher order terms. Notice that the coefficients of the exponential terms $e^{\left(\lambda_{k}+\lambda_{l}\right) t}$ give a measure of the participation of the mode combination $\lambda_{k}+\lambda_{l}$ in a given state variable. Hence, (13) has the potential to be an effective analysis tool for describing modal interactions that involve pairs of system modes. It certainly gives a broader perspective of the system dynamic characteristics than that provided by a linear approximation. In the sequel, the terms "second-order mode" and "combination mode" are used to denote $\lambda_{k}+\lambda_{l}$.

Equation (13) reveals that, if the system is stable, the second term involving $e^{\left(\lambda_{k}+\lambda_{l}\right) t}$ will be more heavily damped than the first-order modes, $e^{\lambda_{k} t}$ or $e^{\lambda_{l} t}$.

\section{A. Key Indices Based on Normal Forms Analysis}

The indices described below have been developed to identify and quantify the extent of nonlinear interactions, and to describe important system characteristics such as mode-state interactions.

Contribution Factors [8]: In the linear case, the Jordan form solution is $y_{j}(t)=y_{j 0} e^{\lambda_{j} t}$, where $y_{j 0}$ is the $j$ th initial condition in the Jordan-form coordinate system. The linear solution in time for the $i$ th state variable is

$$
x_{i}(t)=\sum_{j=1}^{n} u_{i j} y_{j 0} e^{\lambda_{j} t}=\sum_{j=1}^{n} \sigma_{i j} e^{\lambda_{j} t}
$$


The $\sigma_{i j}=u_{i j} y_{j 0}$ are referred to as linear "contribution factors" [20], [21]. They indicate the size of mode $j$ 's contribution to the oscillations of state $i$ for a given disturbance. The solution (14) consists of a sum of weighted exponential oscillations. The "weights" are the contribution factors. The frequencies of oscillation are given by the imaginary parts of the eigenvalues $\left(\lambda_{j}\right)$.

The comparison of the linear and second-order solutions, expressed in (14) and (13) respectively, shows that the secondorder solution contains many more potential frequencies of oscillation. The bracketed term in (13) represents the second-order effects related to these combinations of frequencies from the Jordan form (8). As shown in (13), these effects are transformed to the states $x$ using the right eigenvectors $\left(u_{i j}\right)$.

The second-order solutions given in (13) may be rewritten as

$$
x_{i}(t)=\sum_{j=1}^{n} \sigma_{2 i j} e^{\lambda_{j} t}+\sum_{k=1}^{n} \sum_{l=k}^{n} \sigma_{22 i k l} e^{\left(\lambda_{k}+\lambda_{l}\right) t} .
$$

Here, $\sigma_{2 i j}=u_{i j} z_{j o}$ and $\sigma_{22 i k \ell}=z_{k 0} z_{\ell 0} \sum_{j=1}^{n} u_{i j} h 2_{k \ell}^{j}$. Since the matrices $H^{i}, i=1, \ldots, n$ are symmetric, (13) is often rewritten using the notation $k=1, l=k$ as in (15) above. Thus, the second-order contribution factors are defined in a manner very similar to the linear contribution factors described earlier. They are measures of the size and phase of the oscillations that make up the approximate second-order solution for the states. $\sigma_{2 i j}$ gives the contribution of the single-eigenvalue mode $\left(\lambda_{j}\right)$ to the response of state $i$. Similarly $\sigma_{22 i k l}$ gives the contribution of the combination mode $\left(\lambda_{k}+\lambda_{l}\right)$ to the response of state $i$. (Note that the linear contribution factor $\sigma_{i j}$ constitutes part of the second-order contribution factor $\sigma_{2 i j}$.)

Nonlinear Participation Factors [8]: Linear participation factors, which are defined in [22], are a commonly used measure of mode-state interactions. The participation factor $p_{i j}$ is a measure of the participation of the $i$ th state in the trajectory of the $j$ th mode. One advantage of using participation factors is that they measure mode-state relationships independent of eigenvector scaling. This is because they are functions of both the left and right eigenvectors. In [22], it is observed that the participation factors represent the size of the modal oscillations in a state when only that state is perturbed. Hence, the initial condition vector is $x_{0}=e_{j}$ (all elements of $e_{j}$ are zero except the $j$ th, which is one). This means that when $x_{0}=e_{j}$, the time solution for the $i$ th state variable $x_{i}$ is

$$
x_{i}(t)=\sum_{j=1}^{n} p_{i j} e^{\lambda_{j} t} .
$$

In this type of analysis, the responses for each of the perturbed states are assumed to combine to give the full response.

Using the theory of Normal Forms this concept can be extended to include second-order terms. The approximate secondorder, Normal Form inverse transformation for any initial condition is given by $z_{j}=y_{j}-h 2^{i}(y)$. When the initial condition vector $x_{0}=e_{i}$ is applied, the Jordan form initial conditions become

$$
y_{j 0}=v_{j i} \text {. }
$$

The Normal Form initial conditions, using the second-order approximation of the inverse transformation $z_{j 0}=y_{j 0}$ $\sum_{k=l}^{n} \sum_{l=k}^{n} h 2_{k l}^{j} y_{k 0} y_{l 0}$, are

$$
z_{j 0}=v_{j i}-\sum_{k=1}^{n} \sum_{l=k}^{n} h 2_{k l}^{j} v_{k i} v_{l i}=v_{j i}+v 2_{j i i} .
$$

The solution for the $i$ th state variable (when $x_{j 0}=0$, for all $j \neq i$ ) can be written as

$$
\begin{aligned}
& x_{i}(t)=\sum_{j=1}^{n} u_{i j}\left(v_{j i}+v 2_{j i i}\right) e^{\lambda_{j} t} \\
& \quad+\sum_{k=1}^{n} \sum_{l=k}^{n} u 2_{i k l}\left(v_{k i}+v 2_{k i i}\right)\left(v_{l i}+v 2_{l i i}\right) e^{\left(\lambda_{k}+\lambda_{l}\right) t} .
\end{aligned}
$$

Using the same approach as in the linear case, the second-order participation factors can be defined according to

$$
x_{i}(t)=\sum_{j=1}^{n} p 2_{i j} e^{\lambda_{j} t}+\sum_{k=1}^{n} \sum_{l=k}^{n} p 2_{k l}^{i} e^{\left(\lambda_{k}+\lambda_{l}\right) t}
$$

(when $x_{j 0}=0$ for all $j \neq i$ ). Note that there are two types of second-order participation factors. $p 2_{i j}$ represents the secondorder participation of the $i$ th state in the $j$ th single-eigenvalue mode. These factors can be thought of as providing secondorder corrections to the linear participation factor information. In fact, viewing (19) reveals that the linear participation factor $\left(p_{i j}=u_{i j} v_{j i}\right)$ is one term in the expression for $p 2_{i j}$. The second type of second-order participation factor, $p 2_{k l}^{i}$, represents the second-order participation of the $i$ th state in the "mode" formed by the combination of the eigenvalues $\lambda_{k}$ and $\lambda_{l}$, e.g., by $\left(\lambda_{k}+\right.$ $\lambda_{l}$ ). As in the linear case, these second-order factors are independent of eigenvector scaling.

As previously stated, the inverse transformation is approximate. To obtain a more accurate value for the Normal Form initial conditions, the iterative procedure described in of [23, Ch. 2] was used to solve (12) and find the $z_{j 0}$ 's numerically. This numerical method does not provide an expression for the inverse Normal Form transformation. However, it does provide a method for more accurately determining numerical values for the initial conditions of the Normal Form. Because the participation factors are found by applying a specific initial condition $\left(x_{0}=e_{j}\right)$, the participation factors may also be determined numerically using the iterative approach. When the initial condition vector $x_{0}$ is set equal to $e_{j}$, the contribution factors become the participation factors.

Nonlinearity Index $(I(j))$ [5], [24]: This index provides a measure of the effect of the nonlinear terms in the approximate closed form solution. It compares the solution obtained by using only the linear terms to that obtained by using the secondorder terms, and determines the extent to which the secondorder terms are dominant. This index is evaluated for all the Jordan form variables that are highly excited by the disturbance. Among these variables we then determine the specific variable which has the largest index $I(j)$. A large Index $I(j)$ indicates a high degree of nonlinear effects in a variable and provides an indication of the fundamental mode in the linear term of the variable solution, and its interaction with the modes in the second-order terms of the solution. A high value of $I(j)$ could 
indicate that the second-order terms are significant indicating nonlinear interaction, or could indicate that the difference between $y_{j 0}$ and $z_{j 0}$ is large, also indicating that the second-order terms have significant contributions which affect the behavior of the closed-form solution

$$
I(j)=\frac{\left|\left(y_{j 0}-z_{j 0}\right)+\max _{k, l}\left(h 2_{k l}^{j} z_{k 0} z_{l 0}\right)\right|}{\left|z_{j 0}\right|} .
$$

Nonlinear Interaction Index $(I I(j))$ [5]: This Index determines whether the nonlinear effects arise from the second-order terms indicating a strong modal interaction, or whether the second terms affect the initial solution in the $z$ variables indicating a dominant linear mode

$$
I I(j)=\frac{\max _{k, \ell}\left|h 2_{2 k \ell}^{j} z_{k o} z_{\ell o}\right|}{\left|z_{j o}\right|} .
$$

Nonlinear Modal Persistence Measures: Three measures were defined to quantify the extent of dominance of the various modes in the time solution. These measures are denoted $T_{\text {set }}$, $T_{r}$ and $I I(j) \times T_{r}$ and are defined as follows.

For a combination mode $\lambda_{k}+\lambda_{l}$

$$
T_{\text {set }}=\frac{-4.0}{\operatorname{Real}\left(\lambda_{k}+\lambda_{l}\right)}
$$

$T_{\text {set }}$ provides a measure of the settling time of the combination mode

$$
T_{r}=\frac{\text { Time Constant for Combination mode }\left(\lambda_{k}+\lambda_{l}\right)}{\text { Time Constant for Dominant mode }\left(\lambda_{j}\right)} .
$$

$T_{r}$ is the ratio of the time constants of the combination modes and the dominant mode. A small $T_{r}$ indicates a significant presence of the combination mode.

A relatively high value of the product $I I(j) \times T_{r}$ tends to reveal a strong modal interaction of longer duration. It appears that the index $I I(j) \times T_{r}$ provides a measure of the energy in a modal interaction. This index therefore may be significant in determining if a composite mode is likely to appear in the power-spectral-density or discrete Fourier transform (DFT) of a disturbance recording. This index is referred to as the Composite Mode Energy Index (CMEI).

\section{TEST SySTEM}

The test system selected for this work is shown in Fig. 1. This system is the four-generator system introduced in [14] as a system suitable for the analysis of electromechanical oscillations. This system has subsequently been widely used for studying different issues related to small-signal stability analysis.

The generators are modeled using a two-axis model with one winding in each axis. Each generator is equipped with an AVR with a transient gain reduction of 10 , a fast-response exciter represented by a single time constant and a gain. The loads L1 and L2 are modeled as constant impedances. The data for the system

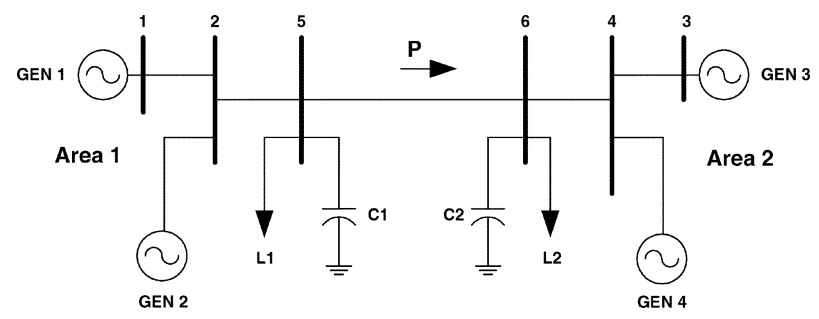

Fig. 1. Two-area test system.

are provided in Appendix B. There is one inter-area mode associated with the oscillations of the two areas, and two local modes associated with the oscillations of the generators within each area.

The selected system operating condition for the study is a highly stressed condition close to voltage collapse, characterized by a tie line flow of $410 \mathrm{MW}$ from Area 1 to Area 2. This operating point was chosen to more readily expose the nonlinear characteristics of the system.

The damping ratio of the inter-area, and the two local modes are $0.04,0.15$, and 0.23 , respectively.

\section{NONLINEAR ModAl ANALYSIS}

Several system conditions were considered in the course of this investigation. The two test cases analyzed in this section were selected to highlight information provided by the secondorder modal analysis. In these test cases the stress is varied by subjecting the system to a three-phase short circuit at Bus 5 and clearing the fault at different times.

Case 1: This case represents a lower stress condition in which the three phase fault applied at Bus 5 is cleared in $0.010 \mathrm{~s}$ with no line switching.

Case 2: This case represents a higher stress condition in which the three phase fault applied at Bus 5 is cleared in $0.019 \mathrm{~s}$ with no line switching.

The system is relatively well damped; the damping ratio of the inter-area, and the two local modes are 0.10, 0.16, and 0.20, respectively.

A systematic presentation related to the computation of the initial conditions, key indices, and a discussion of pertinent issues associated with the application of the Normal Forms analysis follows.

\section{A. Initial Condition for $\mathrm{z}_{0}$}

The initial condition in the Normal Form variables is a critical aspect for the computation of the various indices described in Section II. The initial conditions for the state variables $(x)$ for each test case were obtained using a conventional transient stability program. The following procedure is then followed to obtain the initial conditions in the Normal Form variables via a solution to the equations given in (12).

1. $x_{0}$ : The initial condition $x_{0}=x_{c l}-x_{S E P}$ where $x_{S E P}$ is the post disturbance equilibrium solution and $x_{c l}$ is the system condition at the 
TABLE I

CASE 1 - COMPARISON OF INVERSE $\mathrm{x}_{0}$ SOLUTION

\begin{tabular}{|c|c|c|c|c|}
\hline Var.\# & $\begin{array}{c}x_{0}-\text { Conventional } \\
\text { Time Domain }\end{array}$ & $z_{0}$ - Iterative Algorithm & $y_{0}-$ Inverse Transformation & $\begin{array}{c}x_{0}-\text { Inverse } \\
\text { Transformation }\end{array}$ \\
\hline 1 & -0.02037471 & $0.00543-\mathrm{j} 0.14835$ & $0.04618-\mathrm{j} 0.00817$ & -0.02037471 \\
\hline 2 & -0.01808686 & $0.00543+\mathrm{j} 0.14835$ & $0.04618+\mathrm{j} 0.00817$ & -0.01808686 \\
\hline 3 & 0.00062505 & $-2.75931+\mathrm{j} 0.95981$ & $-0.00436-\mathrm{j} 0.02131$ & 0.00062505 \\
\hline 4 & 0.00237729 & $-2.75931-\mathrm{j} 0.95981$ & $-0.00436+j 0.02131$ & 0.00237729 \\
\hline 5 & 0.01306551 & $0.30507-\mathrm{j} 1.86226$ & $-3.26666-\mathrm{j} 5.12417$ & 0.01306551 \\
\hline 6 & 0.00528722 & $0.30507+\mathrm{j} 1.86226$ & $-3.26666+j 5.12417$ & 0.00528722 \\
\hline 7 & 0.00225042 & $-3.32094-\mathrm{j} 22.98467$ & $50.72027-\mathrm{j} 16.10699$ & 0.00225042 \\
\hline 8 & 0.00686157 & $-3.32094+j 22.98467$ & $50.72027+\mathrm{j} 16.10699$ & 0.00686157 \\
\hline 9 & 0.15920000 & $-19.46105+j 7.14944$ & $-32.14068+j 3.90333$ & 0.15920000 \\
\hline 10 & 0.21000000 & $-19.46105-\mathrm{j} 7.14944$ & $-32.14068-\mathrm{j} 3.90333$ & 0.21000000 \\
\hline 11 & -0.01680000 & $0.36559-\mathrm{j} 2.05989$ & $0.15172-\mathrm{j} 2.15811$ & -0.01680000 \\
\hline 12 & -0.04380000 & $0.36559+\mathrm{j} 2.05989$ & $0.15172+\mathrm{j} 2.15811$ & -0.04380000 \\
\hline 13 & 0.00014222 & $-0.43461-\mathrm{j} 2.41433$ & $-0.44703-\mathrm{j} 1.33013$ & 0.00014222 \\
\hline 14 & -0.00081201 & $-0.43461+j 2.41433$ & $-0.44703+\mathrm{j} 1.33013$ & -0.00081201 \\
\hline 15 & -0.00107874 & -28.43799 & -32.00435 & -0.00107874 \\
\hline 16 & 0.98592352 & -15.39091 & -4.60813 & 0.98592352 \\
\hline 17 & 1.75014994 & -5.06478 & 0.90793 & 1.75014994 \\
\hline 18 & 0.09678894 & -21.09669 & 0.00332 & 0.09678894 \\
\hline 19 & 0.15462569 & -0.12369 & 0.08136 & 0.15462569 \\
\hline 20 & -0.23550000 & -467.92135 & -6.91943 & -0.23550000 \\
\hline 21 & -0.41610000 & 14.17915 & 11.51394 & -0.41610000 \\
\hline 22 & -0.02280000 & -168.88409 & -15.81247 & -0.02280000 \\
\hline 23 & -0.03660000 & 21.24291 & -24.14459 & -0.03660000 \\
\hline 24 & 0.00011524 & 47.70852 & -13.77897 & 0.00011524 \\
\hline 25 & 0.00025150 & -160.95428 & 6.10720 & 0.00025150 \\
\hline 26 & -0.00001311 & -538.21881 & -8.59830 & -0.00001311 \\
\hline 27 & -0.00001474 & 18.08421 & -18.30353 & -0.00001474 \\
\hline
\end{tabular}

end of the disturbance obtained from the transient stability program.

2. $y_{0}: y_{0}=V^{T} x_{0}$, where $V$ is the matrix of left eigenvectors calculated for the post disturbance equilibrium condition.

3. $z_{0}$ : The system of (12) is solved for $z_{0}$ using the Newton-Raphson method as follows:

a. Formulate a nonlinear solution problem of the form $f_{j}(z)=z_{j}+\sum_{k=1}^{n} \sum_{l=k}^{n} h 2_{k l}^{j} z_{k} z_{l}-y_{j}=0$, $j=1,2, \ldots, n$

b. Choose an initial estimate for $z_{0}$. From the analysis on a variety of test systems, the choice of $z_{0}=y_{0}$ provides the most robust results. Initialize the iteration counter: $s=0$.

c. Compute the mismatch function for iteration $\mathrm{s} f_{j}\left(z^{(s)}\right)=z_{j}^{(s)}-y_{j}+\sum_{k=1}^{n} \sum_{l=k}^{n} h 2_{k l}^{j} z_{k}^{(s)} z_{l}^{(s)} j=$ $1,2, \ldots, n$

a. Compute the Jacobian of $f(z)$ at $z^{(s)}$ :

$$
\left[A\left(z^{(s)}\right)\right]=\left[\frac{\partial f}{\partial z}\right]_{z=z(s)} .
$$

e. Compute $\Delta z^{(s)}=-\left[A\left(z^{(s)}\right)\right]^{-1} f\left(z^{(s)}\right)$

$f$. Determine the optimal step length $\mu$ using cubic interpolation or any other appropriate procedure and compute

$$
z^{(s+1)}=z^{(s)}+\mu \Delta z^{(s)}
$$

g. Continue the iterative process until a specified tolerance is met. The value of $z$ when the tolerance is met provides the solution $z_{0}$

For both test cases, $y_{0}$ is used as a starting value for the iterative solution. This choice also has a firm analytical basis. The iterative process is based on (12) which in turn is given by the fundamental Normal Form nonlinear transformation (7). From (12) one observes that the solution to $z_{0}$ is obtained by determining how the nonlinear terms described by $h 2$ affect the $y$-variables. The iterative process described above is continued until the solution converges to a small tolerance. In order to verify the accuracy of the $z_{0}$ solution, an inverse transformation first to the $y$-variables and then to the $x$-variables is performed and the $x_{0}$ solution obtained in this fashion is compared with that obtained from the conventional time domain program. It is important to note that in transforming the Normal Form variables, $z$ to the Jordan space $y$, the approximation $z=y-h 2(y)+o(3)$ is used. Thus the starting values of $z_{j 0}$ can be approximated by a truncated series $z_{j o} \approx y_{j o}-\sum_{k=1}^{n} \sum_{l=1}^{n} h 2_{k l}^{j} y_{k o} y_{l o} \approx y_{j o}, j=1, \ldots, n$. The use of the second-order summations $\sum_{k=1}^{n} \sum_{l=1}^{n} h 2_{k l}^{j} y_{k o} y_{l o}$ appears to be of interest for heavy stress (highly nonlinear behavior). Numerical simulations performed on the two-area system appear to indicate that the improved estimate may result in a reduced number of iterations in the Newton-Raphson approach; the first-order approximation, on the other hand, is easier to implement and gives good results for most operating conditions. Tables I and II show the comparison for Case 1 and Case 2, respectively. The results in these tables clearly indicate that the initial conditions in the Normal Form variables 
TABLE II

CASE 2-COMPARISON OF INVERSE $x_{0}$ SOLUTION

\begin{tabular}{|c|c|c|c|c|}
\hline Var.\# & $\begin{array}{c}x_{0}-\text { Conventional } \\
\text { Time Domain }\end{array}$ & $z_{0}$ - Iterative Algorithm & $y_{0}$ - Inverse Transformation & $\begin{array}{c}x_{0}-\text { Inverse } \\
\text { Transformation }\end{array}$ \\
\hline 1 & -0.03725585 & $0.27048+\mathrm{j} 0.09857$ & $0.07474-\mathrm{j} 0.02343$ & -0.03725585 \\
\hline 2 & -0.02995437 & $0.27048-\mathrm{j} 0.09857$ & $0.07474+\mathrm{j} 0.02343$ & -0.02995437 \\
\hline 3 & 0.00126246 & $0.07160+\mathrm{j} 0.47343$ & $-0.00457-\mathrm{j} 0.04310$ & 0.00126246 \\
\hline 4 & 0.00460752 & $0.07160-\mathrm{j} 0.47343$ & $-0.00457+\mathrm{j} 0.04310$ & 0.00460752 \\
\hline 5 & 0.02215094 & $-5.04258-\mathrm{j} 9.05999$ & $-4.03018-\mathrm{j} 6.95564$ & 0.02215094 \\
\hline 6 & 0.01053573 & $-5.04258+j 9.05999$ & $-4.03018+\mathrm{j} 6.95564$ & 0.01053573 \\
\hline 7 & 0.00415556 & $14.03602+\mathrm{j} 13.45906$ & $67.60442-\mathrm{j} 20.79272$ & 0.00415556 \\
\hline 8 & 0.01296019 & $14.03602-\mathrm{j} 13.45906$ & $67.60442+j 20.79272$ & 0.01296019 \\
\hline 9 & 0.30390000 & $-10.89967+j 4.23656$ & $-43.05058+\mathrm{j} 4.63025$ & 0.30390000 \\
\hline 10 & 0.39820000 & $-10.89967-\mathrm{j} 4.23656$ & $-43.05058-\mathrm{j} 4.63025$ & 0.39820000 \\
\hline 11 & -0.03230000 & $3.39290-\mathrm{j} 1.96725$ & $0.19189-\mathrm{j} 2.82764$ & -0.03230000 \\
\hline 12 & -0.08240000 & $3.39290+\mathrm{j} 1.96725$ & $0.19189+\mathrm{j} 2.82764$ & -0.08240000 \\
\hline 13 & 0.00074222 & $-3.57904-\mathrm{j} 4.80359$ & $-0.59565-\mathrm{j} 1.76984$ & 0.00074222 \\
\hline 14 & -0.00301201 & $-3.57904+j 4.80359$ & $-0.59565+\mathrm{j} 1.76984$ & -0.00301201 \\
\hline 15 & -0.00357874 & 77.65555 & -42.86202 & -0.00357874 \\
\hline 16 & 2.19322352 & 4.24931 & -6.15043 & 2.19322352 \\
\hline 17 & 2.62514994 & -2.20115 & 1.17396 & 2.62514993 \\
\hline 18 & 0.21248894 & -6.75536 & 0.01458 & 0.21248894 \\
\hline 19 & 0.33872569 & -1.04771 & 0.11200 & 0.33872569 \\
\hline 20 & -0.32260000 & 356.46075 & -9.07984 & -0.32260000 \\
\hline 21 & -0.56050000 & 1713.73535 & 15.53547 & -0.56050000 \\
\hline 22 & -0.03090000 & -1956.63607 & -20.70656 & -0.03090000 \\
\hline 23 & -0.04910000 & 4475.85311 & -32.43752 & -0.04910000 \\
\hline 24 & 0.00041524 & -85.28318 & -18.50244 & 0.00041524 \\
\hline 25 & 0.00065150 & 577.68649 & 8.16812 & 0.00065150 \\
\hline 26 & 0.00008689 & 686.91695 & -11.28784 & 0.00008689 \\
\hline 27 & 0.00008526 & -167.65710 & -24.07759 & 0.00008526 \\
\hline
\end{tabular}

are accurately obtained and the inverse solutions to the state variable initial conditions agree closely with those obtained from conventional time simulation.

In [25], a different approach for verifying the validity of the initial condition solution is proposed. This paper also suggests several indices to determine the accuracy of the initial conditions based on time domain simulations.

It is important to note that in order to determine the time evolution of the states using the initial conditions, the responses $x(t)$ should be continuously differentiable, i.e., the instant of time for initial conditions must be chosen such that all limiting action by controllers in the system must have ceased after the clearance of the fault.

The method of Normal Forms can also be applied to unstable systems. In this case, a stable post disturbance equilibrium will not be available. However, the Normal Forms analysis can be conducted at the appropriate unstable equilibrium point using the real Normal Forms approach as demonstrated in [6] and critical qualitative results regarding the trajectory and dynamic performance can be obtained.

\section{B. Normal Form Analysis of Case 1}

The original state variables in terms of the variables $x$ are first represented by a Taylor series expansion approximated to the second order. The fundamental linear modes are then computed. Using the similarity transformation derived from the linear term of the series expansion [3], the state variables $x$ are transformed into the Jordan form characterized by the variables $y$. The Jordan form variables describe the time evolution of the linear modal variables of the system. It is to be noted that in the transformations used in this paper, the $x$-variables are real; however, the $y$ and $z$-variables are complex. The $y$-variables also describe the time evolution of the linear modes as characterized by (8). Using the initial conditions for the Normal Form variables, the indices $I(j)$ and $I I(j)$ [(21) and (22)] are evaluated to determine the extent of the nonlinearity and the existence of important interactions. These are listed in Table III. The following observations are made from the results presented in Table III.

1) Among the oscillatory modes, Modes $(5,6),(1,2),(7,8)$, and $(3,4)$ have the largest Index $I(j)$. This index indicates the extent of the nonlinearity.

2) There are several real modes that also have a high value of Index $I(j)$.

3) The modes identified in observation 1 also have a very high value of Index $I I(j)$ which indicates that there could be some nonlinear interaction.

4) We also note from Table I that among the oscillatory modes, Modes $(7,8),(9,10),(3,4),(13,14),(11,12)$, and $(5,6)$ have the largest $z_{0}$.

These observations suggest that Mode $(5,6)$, should be examined in greater detail because it figures predominantly in all the observations made above. On this basis, the interaction coefficients $\left(h 2_{j k}^{i} z_{j 0} z_{k 0}\right.$ terms) of Mode $(5,6)$ are examined. These coefficients are listed in descending order in Table IV. It is observed that several of the larger interactions involve Mode 5 and other real modes listed in Table III, and modes 7, 8, 9, and 
TABLE III

CASE 1 -NORMAL FORM CALCULATIONS

\begin{tabular}{|c|c|c|c|}
\hline Mode \# & Eigenvalue & Index I & Index II \\
\hline 1,2 & $-1.24 \pm \mathrm{j} 7.73$ & 7.29 & 6.33 \\
\hline 3,4 & $-1.56 \pm \mathrm{j} 7.53$ & 2.82 & 2.83 \\
\hline 5,6 & $-0.196 \pm \mathrm{j} 2.03$ & 8.62 & 6.06 \\
\hline 7,8 & $-1.02 \pm \mathrm{j} 0.949$ & 3.43 & 1.43 \\
\hline 9,10 & $-0.64 \pm \mathrm{j} 0.864$ & 1.27 & 0.64 \\
\hline 11,12 & $-0.257 \pm \mathrm{j} 0.389$ & 0.223 & 0.162 \\
\hline 13,14 & $-0.283 \pm \mathrm{j} 0.362$ & 0.847 & 0.440 \\
\hline 15 & -0.44820 & 0.18 & 0.206 \\
\hline 16 & -3.42818 & 2.29 & 1.59 \\
\hline 17 & -4.11232 & 0.447 & 1.63 \\
\hline 18 & -8.42803 & 1.76 & 0.906 \\
\hline 19 & -8.98760 & 9.69 & 11.35 \\
\hline 20 & -94.56329 & 0.969 & 0.519 \\
\hline 21 & -89.70104 & 21.18 & 20.99 \\
\hline 22 & -94.65189 & 1.42 & 0.543 \\
\hline 23 & -90.09897 & 8.36 & 6.22 \\
\hline 24 & -105.07721 & 4.31 & 3.02 \\
\hline 25 & -105.15562 & 3.79 & 2.76 \\
\hline 26 & -109.34801 & 2.25 & 1.27 \\
\hline 27 & -109.01844 & 8.88 & 6.87 \\
\hline
\end{tabular}

10 among the oscillatory modes. The interactions with other oscillatory modes have smaller interaction coefficients. At the bottom of the table there is a listing of an interaction of mode 5 with itself. This interaction coefficient is again very small indicating a very low level of interaction. The largest interaction among oscillatory modes occurs between Modes 5, 7, and 9 . Close examination of the modes listed in Table III shows that

$$
\begin{aligned}
\operatorname{Imag}(\text { Mode } 7+\text { Mode } 9) & =\operatorname{Imag}(-1.66+\mathrm{j} 1.81) \\
& \cong \operatorname{Imag}(\text { Mode } 5)=-\mathrm{j} 2.03 .
\end{aligned}
$$

We note that there is near resonance among the modes only in terms of the frequency. The practical significance of this interaction is that in some instances modes that are observed in practice, e.g., in ring-down tests, can originate due to the interaction of first-order modes.

The nonlinear modal persistence measures, $T_{\text {set }}, T_{r}$, and $I I(j)^{*} T_{r}$, for the dominant Mode 5 are listed in Table V. The results shown in this table indicate that none of the combination modes have a settling time that is close to one half of the settling time of the dominant Mode $(5,6)$ (i.e., $1 / 2 \times 20.4$ s). The combination modes $(5,5)$ and $(6,6)$ have half the settling time but their interaction coefficients given in Table IV are relatively small. This indicates that there is no significant nonlinear interaction. As a further verification of the modal behavior, a discrete Fourier transform of the spectrum of the tie line real power flow in the four machine system, following the $0.01 \mathrm{~s}$ three phase fault was obtained. The plot is shown in Fig. 2. The frequency spectrum clearly illustrates that Mode 5, which has a frequency of $2.016 \mathrm{rad} / \mathrm{s}$, is the dominant mode. An additional frequency of $4.0212 \mathrm{rad} / \mathrm{s}$ is also present but this frequency does not have a significant magnitude in the spectrum. This is clearly reinforced by the last row in Table IV where the double frequency mode has a very small interaction coefficient.

All other frequencies do not have significant contributions in the spectrum. This spectral analysis also reinforces the Normal
TABLE IV

CASE 1 -InTERACTION CoEFFICIENTS For Mode $(5,6)$

\begin{tabular}{|c|c|c|}
\hline Interaction Coefficient & $\boldsymbol{j}$ & $\boldsymbol{k}$ \\
\hline 6.06430 & 18 & 18 \\
\hline 2.85266 & 16 & 18 \\
\hline 2.44078 & 7 & 9 \\
\hline 1.90739 & 17 & 18 \\
\hline 1.60348 & 7 & 8 \\
\hline 1.54008 & 5 & 8 \\
\hline 1.53598 & 5 & 7 \\
\hline 1.47709 & 7 & 10 \\
\hline 1.44963 & 9 & 10 \\
\hline 1.43296 & 9 & 18 \\
\hline 1.43076 & 8 & 9 \\
\hline 1.40848 & 5 & 9 \\
\hline 1.26243 & 5 & 10 \\
\hline 1.24113 & 9 & 9 \\
\hline 1.18460 & 7 & 7 \\
\hline 1.12316 & 3 & 18 \\
\hline 1.07860 & 7 & 18 \\
\hline 1.04456 & 13 & 18 \\
\hline 1.01153 & 14 & 18 \\
\hline 0.99076 & 16 & 17 \\
\hline 0.98856 & 5 & 15 \\
\hline 0.95793 & 4 & 18 \\
\hline 0.93702 & 10 & 18 \\
\hline-------- & --------- & ------ \\
\hline $\mathbf{0 . 2 1 0 8 1}$ & $\mathbf{5}$ & $\mathbf{5}$ \\
\hline & & \\
\hline
\end{tabular}

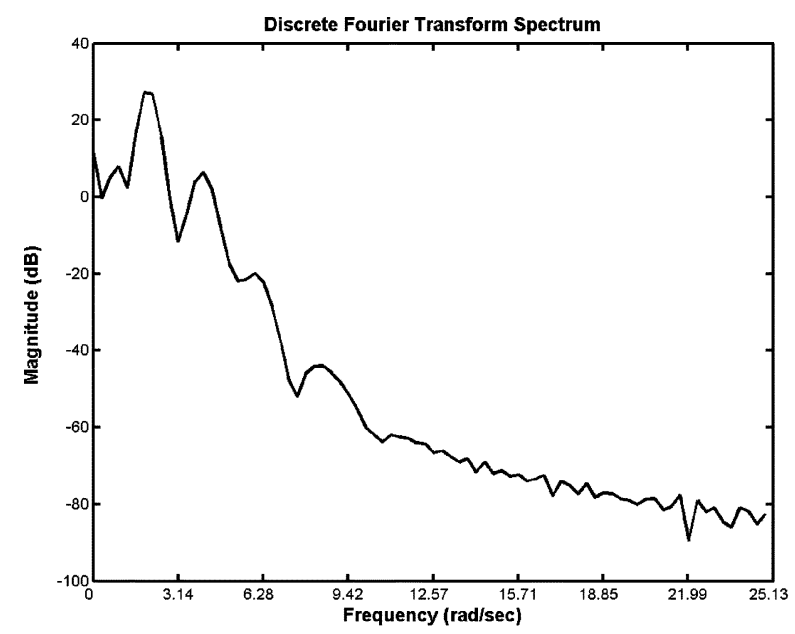

Fig. 2. Case 1: frequency spectrum.

Form analysis results that indicate that for the clearing time of $0.01 \mathrm{~s}$ the test system does not demonstrate significant nonlinear interaction. The dynamics are dominated by the inter area Mode $(5,6)$.

\section{Normal Form Analysis of Case 2}

The Normal Form analysis for the more severely stressed Case 2 is described in this section. Table VI summarizes the Normal Form calculations. The following observations can be made from the results presented in Table VI. 
TABLE V

Case 1-Quantitative Measures of Combination Mode Dominance for Fundamental Mode (5, 6)

\begin{tabular}{|c|c|c|c|c|c|}
\hline $\boldsymbol{j}$ & $\boldsymbol{k}$ & $\boldsymbol{\lambda}_{\boldsymbol{j}+\boldsymbol{\lambda}_{\boldsymbol{k}}}$ & $\mathbf{T}_{\text {set }}$ & $\mathbf{T}_{\mathbf{r}}$ & $\boldsymbol{I I}_{\mathbf{( j )}} \boldsymbol{*}_{\mathbf{r}}$ \\
\hline 18 & 18 & -16.8561 & 0.2373 & 0.0117 & 0.0708 \\
\hline 16 & 18 & -11.8562 & 0.3374 & 0.0166 & 0.1006 \\
\hline 7 & 9 & $-1.6624+\mathbf{j} 1.8125$ & 2.4061 & 0.1183 & 0.7177 \\
\hline 17 & 18 & -12.5404 & 0.3190 & 0.0157 & 0.0951 \\
\hline 7 & 8 & -2.0418 & 1.9590 & 0.0964 & 0.5843 \\
\hline 5 & 8 & $-1.2177+\mathbf{j} 1.0839$ & 3.2850 & 0.1616 & 0.9798 \\
\hline 5 & 7 & $-1.2177+\mathbf{j} 2.9813$ & 3.2850 & 0.1616 & 0.9798 \\
\hline 7 & 10 & $-1.6624+\mathbf{j} 0.0848$ & 2.4061 & 0.1183 & 0.7177 \\
\hline 9 & 10 & -1.2830 & 3.1177 & 0.1533 & 0.9299 \\
\hline 9 & 18 & $-9.0695+\mathbf{j} 0.8639$ & 0.4410 & 0.0217 & 0.1315 \\
\hline 8 & 9 & $-1.6624-\mathbf{j} 0.0848$ & 2.4061 & 0.1183 & 0.7177 \\
\hline 5 & 9 & $-0.8382+\mathbf{j} 2.8965$ & 4.7720 & 0.2347 & 1.4233 \\
\hline 5 & 10 & $-0.8382+\mathbf{j} 1.1688$ & 4.7720 & 0.2347 & 1.4233 \\
\hline 9 & 9 & $-1.2830+\mathbf{j} 1.7277$ & 3.1177 & 0.1533 & 0.9299 \\
\hline 7 & 7 & $-2.0418+\mathbf{j} 1.8974$ & 1.9590 & 0.0964 & 0.5843 \\
\hline 3 & 18 & $-9.9883+\mathbf{j} 7.5276$ & 0.4005 & 0.0197 & 0.1194 \\
\hline 7 & 18 & $-9.4490+\mathbf{j} 0.9487$ & 0.4233 & 0.0208 & 0.1263 \\
\hline 13 & 18 & $-8.7107+\mathbf{j} 0.3617$ & 0.4592 & 0.0226 & 0.1370 \\
\hline 14 & 18 & $-8.7107-\mathrm{j} 0.3617$ & 0.4592 & 0.0226 & 0.1370 \\
\hline 16 & 17 & -7.5405 & 0.5305 & 0.0261 & 0.1582 \\
\hline 5 & 15 & $-0.6449+\mathbf{j} 2.0326$ & 6.2021 & 0.3051 & 1.8499 \\
\hline 4 & 18 & $-9.9883-\mathbf{j} 7.5276$ & 0.4005 & 0.0197 & 0.1194 \\
\hline 10 & 18 & $-9.0695-\mathbf{j} 0.8639$ & 0.4410 & 0.0217 & 0.1315 \\
\hline-------- \\
\hline $\mathbf{5}$ & ------- & $\mathbf{3 . 0 3 2 2}$ \\
\hline
\end{tabular}

TABLE VI

CASE 2-Normal Form CALCUlations

\begin{tabular}{|c|c|c|c|}
\hline Mode \# & Eigen Value & $\boldsymbol{I}$ & $\boldsymbol{I}$ \\
\hline 1,2 & $-1.24 \pm \mathrm{j} 7.73$ & 3.11 & 3.58 \\
\hline 3,4 & $-1.56 \pm \mathrm{j} 7.53$ & 2.79 & 2.96 \\
\hline 5,6 & $-0.196 \pm \mathrm{j} 2.03$ & 2.68 & 2.70 \\
\hline 7,8 & $-1.02 \pm \mathrm{j} 0.949$ & 7.76 & 5.46 \\
\hline 9,10 & $-0.64 \pm \mathrm{j} 0.864$ & 5.53 & 2.85 \\
\hline 11,12 & $-0.257 \pm \mathrm{j} 0.389$ & 1.70 & 0.862 \\
\hline 13,14 & $-0.283 \pm \mathrm{j} 0.362$ & 1.59 & 0.90 \\
\hline 15 & -0.44820 & 2.85 & 1.30 \\
\hline 16 & -3.42818 & 7.27 & 4.83 \\
\hline 17 & -4.11232 & 3.28 & 2.55 \\
\hline 18 & -8.42803 & 2.56 & 1.55 \\
\hline 19 & -8.98760 & 2.51 & 1.40 \\
\hline 20 & -94.56329 & 1.59 & 2.62 \\
\hline 21 & -89.70104 & 2.27 & 1.91 \\
\hline 22 & -94.65189 & 1.95 & 1.02 \\
\hline 23 & -90.09897 & 2.75 & 1.80 \\
\hline 24 & -105.07721 & 3.60 & 2.82 \\
\hline 25 & -105.15562 & 1.39 & 1.15 \\
\hline 26 & -109.34801 & 2.56 & 1.55 \\
\hline 27 & -109.01844 & 0.774 & 1.47 \\
\hline
\end{tabular}

1. All the oscillatory modes have a high interaction index. Among the oscillatory modes, Modes $(7,8),(9,10),(1$, $2),(3,4)$, and $(5,6)$, have the largest index $I$. The other modes, Modes $(11,12)$, and $(13,14)$, have index $I$ that are comparable in magnitude

2. Among the oscillatory modes, Modes $(7,8),(9,10),(5,6)$, $(13,14)$, and $(11,12)$, have the largest $z_{0}$.

Some of the real modes also have large $I$ and significantly large $z_{0}$. These include Modes 16, 15, 17, and 18. Some of the other real modes that have these characteristics are $20,21,22$, $23,24,25,26$, and 27; however, these modes have very short time constants (about $0.01 \mathrm{~s}$ ).

The indices listed in Tables III and VI can only be used to compare the modes for individual cases. They cannot be used as a measure to compare modes between two different cases. This is because these indices use normalized eigenvectors and the normalization differs between cases.

Based on these observations it appears as if Mode $(3,4)$, Mode $(7,8)$, Mode $(1,2)$, Mode $(5,6)$, and Mode $(9,10)$ among the oscillatory modes and real Mode $15,16,17$, and 18 should all be examined. The interaction coefficients of these modes were examined in detail. Lack of space prevents listing all interaction coefficients. However, the system dynamics clearly indicate that the interaction is dominated by large interaction coefficients between these modes and Mode $(5,6)$ together with other modes. Another salient feature is that several of these modes have large interaction coefficients with the combination Mode $(5,5)$. This analysis indicated that system dynamic performance will be dominated by Mode $(5,6)$. In addition, in this case which is highly stressed by the disturbance, the combination Mode (5, 5 ) has significantly higher interactions with all other modes.

Table VII provides the interaction coefficients for the dominant Mode $(5,6)$. It is important to note that the combination Mode $(5,5)$ has strong interaction with Mode $(5,6)$ unlike the situation in Case 1, indicating the presence of significant nonlinear interaction.

The quantitative indices for Mode $(5,6)$ for Case 2 are shown in Table VIII. These results again clearly illustrate that the linear Mode $(5,6)$ interacts with several other modes and the combination of these modes have settling times that are nearly half the 
TABLE VII

CASE 2-InTERACTION COEFFICIENTS FOR Mode $(5,6)$

\begin{tabular}{|c|c|c|}
\hline Interaction Coefficient & $j$ & $\boldsymbol{k}$ \\
\hline 2.69947 & 5 & 15 \\
\hline 2.28727 & 5 & 6 \\
\hline 1.28960 & 5 & 8 \\
\hline 1.28617 & 5 & 7 \\
\hline 1.20454 & 5 & 11 \\
\hline 1.19738 & 5 & 12 \\
\hline 1.15830 & 5 & 5 \\
\hline 0.79444 & 5 & 9 \\
\hline 0.71206 & 5 & 10 \\
\hline 0.58800 & 5 & 14 \\
\hline 0.53917 & 6 & 7 \\
\hline 0.45337 & 5 & 13 \\
\hline 0.38376 & 6 & 6 \\
\hline 0.35464 & 6 & 8 \\
\hline 0.31152 & 6 & 15 \\
\hline 0.29017 & 7 & 15 \\
\hline 0.25231 & 6 & 9 \\
\hline 0.20980 & 7 & 9 \\
\hline 0.20462 & 7 & 8 \\
\hline 0.17228 & 11 & 15 \\
\hline 0.15920 & 7 & 11 \\
\hline 0.15889 & 8 & 15 \\
\hline 0.15665 & 6 & 10 \\
\hline 0.15372 & 11 & 12 \\
\hline 0.15303 & 13 & 14 \\
\hline
\end{tabular}

settling time of the fundamental mode. In addition a salient feature of Case 2 is that the combination Mode $(5,5)$ and Mode (6, 6 ), which have the same frequency, clearly show a marked presence and their settling times are exactly half that of the dominant mode. This indicates a strong presence of nonlinear modal interaction and reinforces the presence of a combination frequency in the dynamic response of the system. The DFT of the tie line power flow for Case 2, shown in Fig. 3, verifies this observation.

The frequency spectrum for Case 2 shown in Fig. 3 corroborates the inference derived from the Normal Form analysis. It shows that the frequency spectrum of the tie line real power flow is dominated by the fundamental Mode $(5,6)$. In addition, for Case 2, the combination Mode $(5,5)$, which has twice the frequency of the fundamental mode, is present to a significant extent indicating that a combination frequency does exist in the dynamics observed in time domain. This observation is also supported by the Normal Form analysis that was presented above for Case 2.

\section{Discussion}

The results presented above for a simple test system demonstrate that the increase in stress caused by the severity of the fault does result in nonlinear interaction of the fundamental modes. The analysis conducted using the method of Normal Forms identifies this interaction correctly. The modes involved in the interaction can also be determined. The validity of the interaction is also verified by conducting spectral analysis on the time domain variation of the tie line real power flow. The

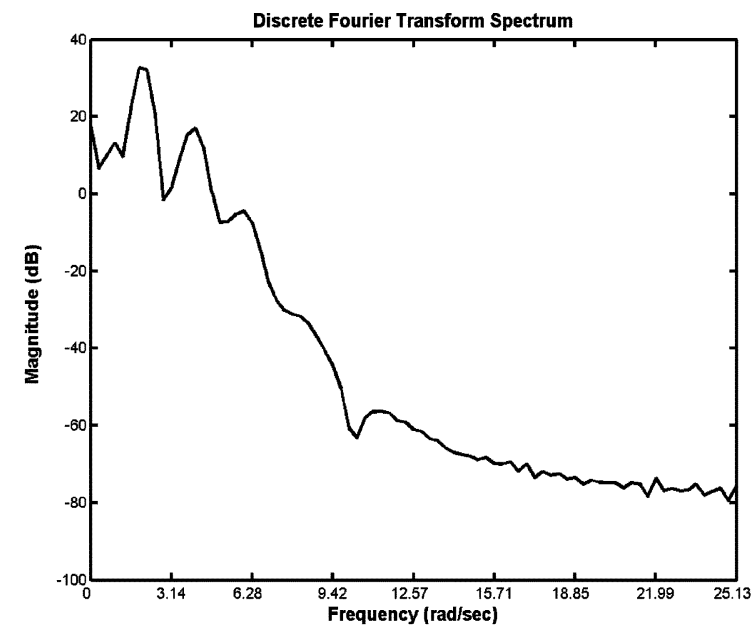

Fig. 3. Case 2: frequency spectrum.

Normal Form analysis has the ability to distinguish between the degrees of interaction when different levels of stress are considered.

This has been demonstrated by the analysis conducted on the two cases with varying levels of stress. In Case 1, the analysis points out that there is no significant interaction among the modes, whereas in Case 2, the more stressed case, the analysis correctly identifies the nonlinear interaction. This is again confirmed from the spectral analysis which shows that the $(5,5)$ combination mode has a higher participation in the frequency spectrum of the stressed case. It should also be noted that the interaction coefficients for the dominant mode, Mode $(5,6)$, are much higher in Case 2. This is especially true for a combination of Mode 5 with itself and another oscillatory mode.

This observation can be made by comparing the results in Tables IV and VII. Comparing the results in Tables V and VIII it is noted that Case 2 has several combination modes that have significant settling time in comparison to the dominant Mode $(5,6)$. These modes also have large product terms $I I(j) \times T_{r}$ indicating the persistence of strong modal interaction. There are a few such terms in Case 1, however, these combination modes do not have a long settling time or ratio of the time constant.

Some of the specific questions listed in Section I, addressed by this analysis include the following:

1) Practical Significance: The analysis shows that nonlinear modal interactions are an inherent characteristic of power system dynamic phenomena. They occur as a result of two important aspects of the dynamic phenomena: 1) the post-disturbance network characteristics and 2) the impact of the disturbance. The modal interaction depends on the stress in the system. The interaction can be determined using the method of Normal Forms. In addition, the analysis can distinguish between the levels of interaction in differently stressed analysis cases.

Related work reported in [21], [25], and [26] show another characteristic of practical significance associated with Normal Form analysis. In these references, transient responses are shown for linear analysis, Normal Form analysis, and step-by-step transient stability analysis for both less stressed and heavily stressed systems. The results obtained indicate 
TABLE VIII

Case 2-Quantitative Measures of Combination Mode Dominance for Fundamental Mode $(5,6)$

\begin{tabular}{|c|c|c|c|c|c|}
\hline $\boldsymbol{j}$ & $\boldsymbol{k}$ & $\boldsymbol{\lambda}_{\boldsymbol{j}+\boldsymbol{\lambda}_{\boldsymbol{k}}}$ & $\mathbf{T}_{\text {set- }}$ & $\mathbf{T}_{\mathbf{r}}$ & $\left.\boldsymbol{I I}_{\boldsymbol{j}}\right)^{*} \mathbf{T}_{\mathbf{r}}$ \\
\hline 5 & 15 & $-0.6449+\mathrm{j} 2.0326$ & 6.2021 & 0.3051 & 0.8235 \\
\hline 5 & 6 & -0.3935 & 10.1657 & 0.5000 & 1.3497 \\
\hline 5 & 8 & $-1.2177+\mathrm{j} 1.0839$ & 3.2850 & 0.1616 & 0.4362 \\
\hline 5 & 7 & $-1.2177+\mathrm{j} 2.9813$ & 3.2850 & 0.1616 & 0.4362 \\
\hline 5 & 11 & $-0.4536+\mathrm{j} 2.4217$ & 8.8180 & 0.4337 & 1.1708 \\
\hline 5 & 12 & $-0.4536+\mathrm{j} 1.6436$ & 8.8180 & 0.4337 & 1.1708 \\
\hline $\mathbf{5}$ & $\mathbf{5}$ & $\mathbf{- 0 . 3 9 3 5}+\mathbf{j} 4 \mathbf{0 6 5 2}$ & $\mathbf{1 0 . 1 6 5 7}$ & $\mathbf{0 . 5 0 0 0}$ & $\mathbf{1 . 3 4 9 7}$ \\
\hline 5 & 9 & $-0.8382+\mathrm{j} 2.8965$ & 4.7720 & 0.2347 & 0.6336 \\
\hline 5 & 10 & $-0.8382+\mathrm{j} 1.1688$ & 4.7720 & 0.2347 & 0.6336 \\
\hline 5 & 14 & $-0.4794+\mathrm{j} 1.6709$ & 8.3432 & 0.4104 & 1.1078 \\
\hline 6 & 7 & $-1.2177-\mathrm{j} 1.0839$ & 3.2850 & 0.1616 & 0.4362 \\
\hline 5 & 13 & $-0.4794+\mathrm{j} 2.3943$ & 8.3432 & 0.4104 & 1.1078 \\
\hline $\mathbf{6}$ & $\mathbf{6}$ & $\mathbf{- 0 . 3 9 3 5 - j 4 . 0 6 5 2}$ & $\mathbf{1 0 . 1 6 5 7}$ & $\mathbf{0 . 5 0 0 0}$ & $\mathbf{1 . 3 4 9 7}$ \\
\hline 6 & 8 & $-1.2177-\mathrm{j} 2.9813$ & 3.2850 & 0.1616 & 0.4362 \\
\hline 6 & 15 & $-0.6449-\mathrm{j} 2.0326$ & 6.2021 & 0.3051 & 0.8235 \\
\hline 7 & 15 & $-1.4691+\mathrm{j} 0.9487$ & 2.7227 & 0.1339 & 0.3615 \\
\hline 6 & 9 & $-0.8382-\mathrm{j} 1.1688$ & 4.7720 & 0.2347 & 0.6336 \\
\hline 7 & 9 & $-1.6624+\mathrm{j} 1.8125$ & 2.4061 & 0.1183 & 0.3195 \\
\hline 7 & 8 & -2.0418 & 1.9590 & 0.0964 & 0.2601 \\
\hline 11 & 15 & $-0.7051+\mathrm{j} 0.3891$ & 5.6731 & 0.2790 & 0.7532 \\
\hline 7 & 11 & $-1.2778+\mathrm{j} 1.3378$ & 3.1304 & 0.1540 & 0.4156 \\
\hline 8 & 15 & $-1.4691-\mathrm{j} 0.9487$ & 2.7227 & 0.1339 & 0.3615 \\
\hline 6 & 10 & $-0.8382-\mathrm{j} 2.8965$ & 4.7720 & 0.2347 & 0.6336 \\
\hline 11 & 12 & -0.5138 & 7.7857 & 0.3829 & 1.0337 \\
\hline 13 & 14 & -0.5654 & 7.0749 & 0.3480 & 0.9394 \\
\hline
\end{tabular}

that the method of Normal Forms accurately captures the time evolution of the state variables even in highly stressed cases. The significance of these results resides in further illustrating the model fidelity captured by the higher order formulation allowing for a reliable qualitative description.

Another practical implication of the Normal Form analysis is the identification of higher order resonances or near higher order resonances. In this instance the coefficients of the appropriate higher order terms $h k$ will become very large as indicated by (8) in the case of the second-order term $h 2$. The resulting transients may result in hard limits on the controllers coming into play and as a consequence the resonance condition could be modified or eliminated.

The analysis based on the ratio $\left(T_{r}=\right.$ decay time constant of mode $\left(\lambda_{k}+\lambda_{l}\right)$ decay time constant of dominant mode) reveals that, even under highly stressed conditions, the transients due to modal interactions decay away in less than half the time it takes for the dominant mode (assumed to be the most lightly damped electro-mechanical mode) to decay. It is important to note the practical significance of this result for the identification of the first-order modes using disturbance monitoring or Prony Analysis, i.e., the following.

a) Disturbance monitoring captures the transient behavior of the system following a major disturbance. For a highly stressed system, the latter half of the transient (after limiting action has ceased) will be free of interacting modes.

b) Ring-down tests are usually carried out under less-stressed conditions and the response can be assumed to be due to the eigenvalues of the linearized system for a period probably well before one half the settling time of the dominant mode. Prony Analysis is thus applicable in this later part of the transient.

2) Use of Higher Order Analysis: Higher order analysis should be considered, if feasible when conventional linear analysis does not provide sufficient accuracy or when the analysis of measured data (e.g., spectral analysis) indicates the presence of phenomena not observable by linear analysis.

3) Engineering Insight: The Normal Form analysis identifies the specific modes involved in the nonlinear interaction. In addition, several indices, with a firm basis in linear analysis, reveal important characteristics of the interacting modes. These indices can be used to determine appropriate control actions to, if necessary, ameliorate the effects of such modes. These indices can also identify the onset of complex dynamic phenomena.

4) Complementing Linear Analysis: Linear Analysis tools have found wide spread use and are well established. When analysis as described in question 2 above, indicates the presence of higher order interaction, second or higher order analysis used as a complement to linear analysis tools would significantly enhance the understanding of the structural basis of the dynamic phenomena and aid in designing appropriate control measures.

5) Computational Burden: Higher order analysis does result in additional computational burden. This consists of the evaluation of the quantities associated with the higher order terms. The computational efficiency can be significantly enhanced by the use of tools like spectral analysis and Prony analysis [27], [28] to identify the important frequencies and then selectively computing the higher order terms associated with only the interacting modes. The development of algorithms suitable for the Normal Forms analysis of large power systems is an area of research. 
6) Range of Applicability of Second-Order Analysis: This issue has not been addressed in significant depth. A logical approach would be to include terms higher than second order and if significant higher order nonlinear interaction was identified then the analysis would have to be extended to include terms higher than second order.

\section{CONCLUSIONS}

The investigations conducted by the task force have examined several critical issues related to the inclusion of higher order terms for small-signal analysis. The detailed analysis conducted on a sample test system chosen by the task force has provided several critical insights into nonlinear power system dynamic performance. The cases that were analyzed enable the following conclusions to be drawn.

\section{A. Practical Outcomes of Normal Form Analysis and Their Significance}

- Nonlinear modal interaction between fundamental modes of oscillation does occur in power systems. The degree of this interaction is amplified as the system stress increases.

- The degree of stress is a function of 1) the post disturbance operating conditions and 2) the impact of the disturbance. The stress can be increased by either changing the post disturbance operating condition or by changing the severity of the disturbance.

- The method of Normal Forms can accurately detect the onset of significant nonlinear modal interaction and identify the interacting modes.

- Several quantitative indices developed based on the Normal Form analysis quantify the degree of interaction and provide important information regarding the interacting modes, the states participating in these modes, and the degree of the nonlinear interaction. Future work in the area could consist of seeking indices that will improve the accuracy of the analysis.

- The task force in the course of its investigation has also developed several new modal interaction indices that relate the modal interactions with the time response of the system. These indices capture the settling time of the modal combinations and also provide a comparison of the time response of the combination modes to the dominant fundamental mode of oscillation.

- The method of Normal Forms provides engineering insight not available in conventional linear analysis. This has been demonstrated in the analysis provided in Appendix B dealing with the siting of controllers in a case where significant nonlinear interaction is identified. The analysis in this Appendix indicates a location for a conventional PSS that provides better damping than the location suggested by conventional analysis.

- In the analysis conducted, the Normal Form approach uses terms up to second order. The method however, can be extended without loss of generality to include higher order terms. This would significantly increase the computational burden.
- Several techniques have been developed for detecting modal content from time responses [27], [28]. When these techniques identify modal frequencies, not obtained by small-signal linear analysis, Normal Forms analysis could be used to determine the order of the modal interaction.

- The analysis of modal content of the time responses in the cases considered have verified the interaction predicted by the method of Normal Forms. In the stressed case developed in Section IV, the second-order interaction predicted by the method of Normal Forms is also identified by the spectral analysis conducted on the time response. In this case, the interaction happens to be a double frequency mode of the dominant fundamental mode. No such mode exists in linear analysis. The method of Normal Forms however, predicts such an interaction and correctly identifies it as a double-frequency interaction.

- Modes identified from time responses could occur as a result of nonlinear modal interaction. They are not necessarily the modes obtained using linear analysis.

- The method of Normal Forms provides significant information regarding the nonlinear structural characteristics of the system. In cases where strong nonlinear interaction between modes is predicted, the approach can effectively complement the conventional linear analysis tools.

- In recent years, several techniques for monitoring power system events have been developed and implemented. The task force has not verified whether nonlinear modal interactions have been observed in these measurements. The analysis conducted on the modal content of the time responses from simulation, however, do indicate that such interactions may exist. Actual measurements conducted on highly stressed systems should be analyzed and modal interaction if present could be verified using the method of Normal Forms.

\section{B. Shortcomings and Limitations of the Normal Form Analysis}

- The computational burden of the Normal Form analysis is large. Inclusion of even second-order terms for a large system could impose a significant computational burden. As a result, techniques need to be developed to identify interactions from either measurements or time domain simulations and use this information to evaluate only the necessary terms in the Normal Form analysis to reduce the computational burden.

- The calculation of the initial conditions for the Normal Form variables is a highly nonlinear numerical problem. Care should be taken to obtain the solution and practical techniques to verify the suitability of the solution should be developed.

- Several of the indices developed to analyze the system dynamic performance involve the normalized eigen vectors. Since the normalization is case dependent the indices determined for different cases cannot be directly compared. Some efforts to develop directly comparable indices have been made [12], [13], but further work is necessary.

- Terms higher than second order have not yet been included in the Normal Form analysis. Criteria to determine 
TABLE IX

OSCILLATORY MODES

\begin{tabular}{c|c|c|c|c}
\hline $\begin{array}{c}\text { Mode } \\
\#\end{array}$ & Eigenvalue & $\begin{array}{c}\text { Freq. } \\
(\mathrm{Hz})\end{array}$ & $\begin{array}{c}\text { Damping } \\
\text { Ratio }(\%)\end{array}$ & $\begin{array}{c}\text { Dominant } \\
\text { States }\end{array}$ \\
\hline 1,2 & $-1.154 \pm \mathrm{j} 7.72$ & 1.229 & 14.79 & Local, Area $1\left(\delta_{l}, \delta_{2}\right)$ \\
\hline 3,4 & $-1.802 \pm \mathrm{j} 7.53$ & 1.199 & 23.27 & Local, Area $2\left(\delta_{3}, \delta_{4}\right)$ \\
\hline 5,6 & $-3.768 \pm \mathrm{j} 0.38$ & 0.060 & 99.50 & $E_{q l}^{\prime}, E_{q 2}^{\prime}, E_{q 3}^{\prime}, E_{q 4}^{\prime}$ \\
\hline 7,8 & $-1.483 \pm \mathrm{j} 2.09$ & 0.332 & 57.92 & $E_{d 4}^{\prime}, \omega_{4}, \delta_{4}, \omega_{3}, \delta_{3}$ \\
\hline 9,10 & $-0.058 \pm \mathrm{j} 1.31$ & 0.209 & 4.40 & Inter-area $\left(\delta_{l}, \delta_{2}, \delta_{3}, \delta_{4}\right)$ \\
\hline 11,12 & $-1.047 \pm \mathrm{j} 0.65$ & 0.103 & 85.08 & Controls unit GEN4 \\
\hline 13,14 & $-0.291 \pm \mathrm{j} 0.48$ & 0.077 & 51.51 & Controls unit GEN1 \\
\hline 15,16 & $-0.324 \pm \mathrm{j} 0.48$ & 0.076 & 56.27 & Controls unit GEN3 \\
\hline
\end{tabular}

when such terms are needed should be developed and the range of applicability of each higher order term should be developed. The computational burden involved in incorporating such terms should also be carefully investigated

\section{APPENDIX A}

\section{LINEAR VERSUS NONLINEAR ANALYSIS -A BRIEF COMPARISON}

The objective of Appendix A is to illustrate the differences and similarities in the analysis results that arise as a consequence of applying either linear analysis techniques or the Normal Forms method. It is not the purpose of Appendix A to perform extensive linear and nonlinear analyzes of the test system rather; its emphasis is on highlighting a selected number of results that show that the linear and nonlinear methods may offer different perspectives on the system dynamic characteristics. A more comprehensive discussion can be found in [29].

The operating condition for the system is characterized by a tie line flow of $410 \mathrm{MW}$ from Area 1 to Area 2. The exciter gains, generator damping coefficients and loading conditions differ from those used in Section IV and are given in Appendix C. For the selected conditions the system is highly stressed and close to voltage collapse.

\section{A. Linear Analysis}

Table IX gives the system oscillatory modes, their frequencies, and damping ratios. Also included in the table are the dominant states associated with the individual modes computed using linear participation factors [22].

Fig. 4 shows the speed mode shape for the inter-area mode [30]; the orientation of the mode shapes reflects the highly stressed system conditions. Fig. 5 shows the magnitudes of the rotor speed participation factors; the values are normalized with respect to the largest component. For this stressed operating condition, the speed mode shape shows that the four machines swing more-or-less in-phase; a similar mode shape is reported for this system in [14] for cases involving 400-MW power transfers.

The results obtained by linear analysis are limited (by definition) to the information provided by the system eigenvalues and eigenvectors. Consequently, a question of practical importance is "Are there circumstances where it would be valuable to have

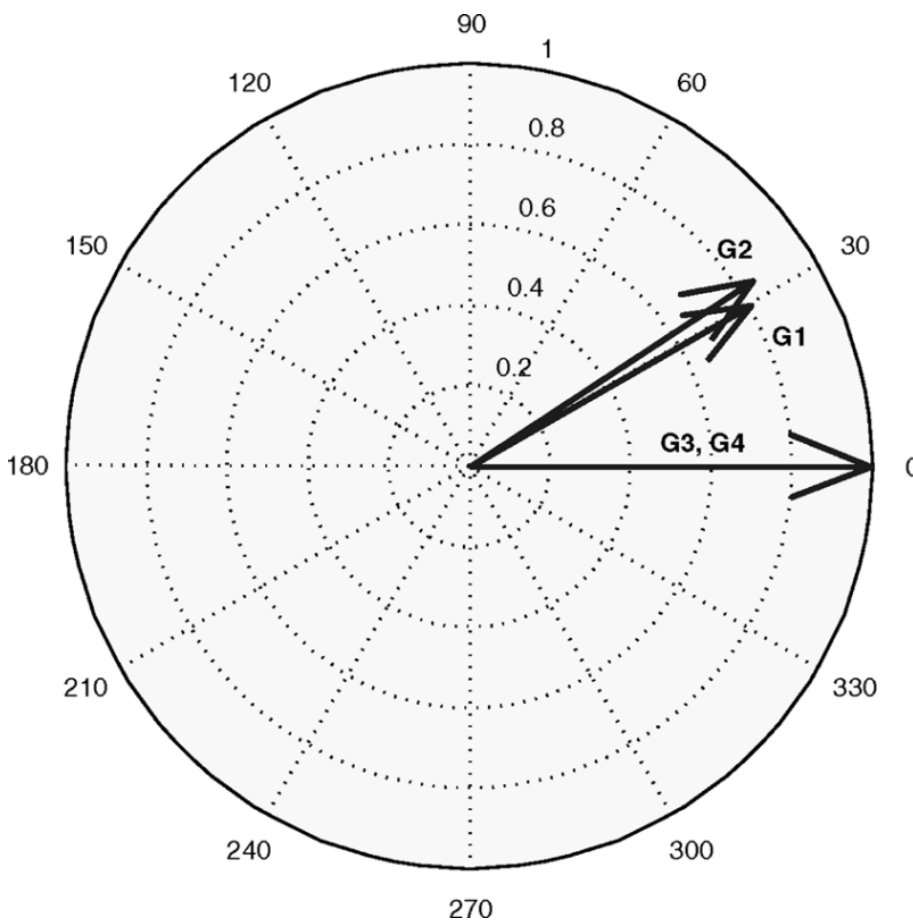

Fig. 4. Mode shapes of rotor speeds for mode 9.

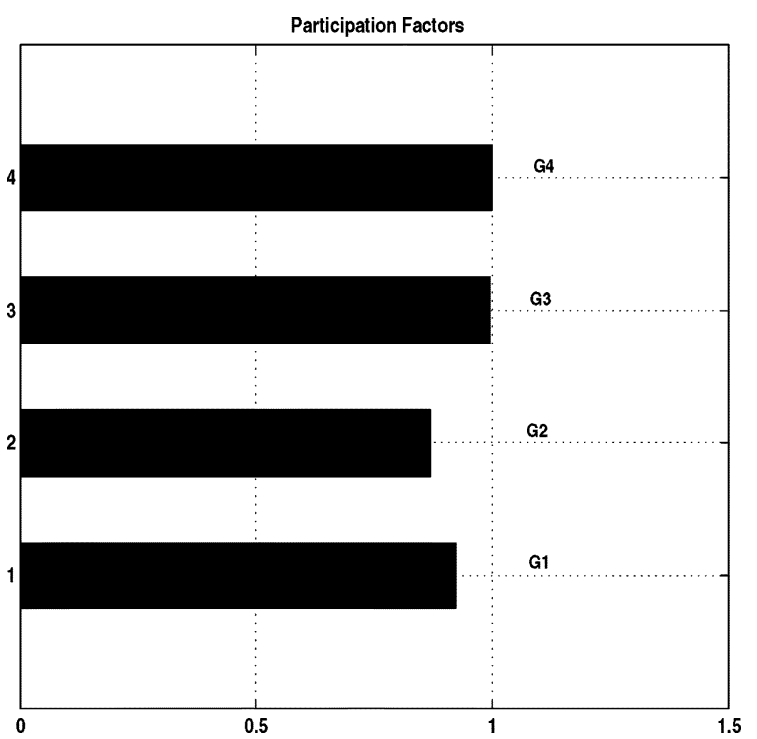

Fig. 5. Speed-based linear participation factors for mode 9 .

access to a more detailed analytical description of the system dynamic characteristics?" An affirmative answer to this question is suggested by the identification of the modes present in the test system response. This type of analysis is typically performed to determine the linear modes present in a signal.

The identification of modes in the inter-area power flow, following a fault at Bus 5, is illustrated in Figs. 6-8. Fig. 6 shows the system response approximately $10 \mathrm{~s}$ after the removal of the fault (the data points in the plot have been shifted to start at time zero). This figure compares the deviation from steady state of the inter-area power flow, $\Delta \mathrm{P}$, computed by a transient stability program, and the response of an identified system computed using the ERA method [31]. The identified system consists of 


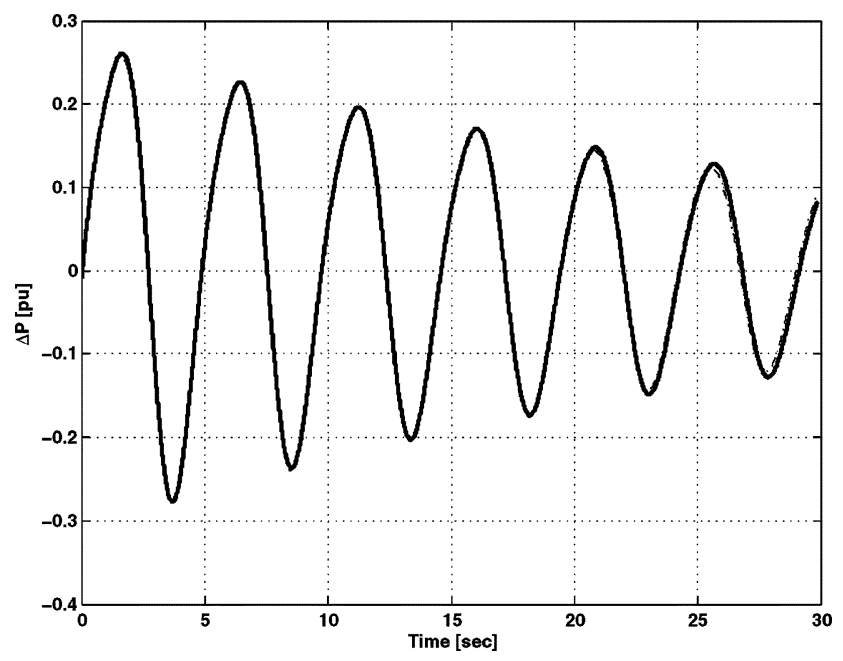

Fig. 6. Inter-area power flow.

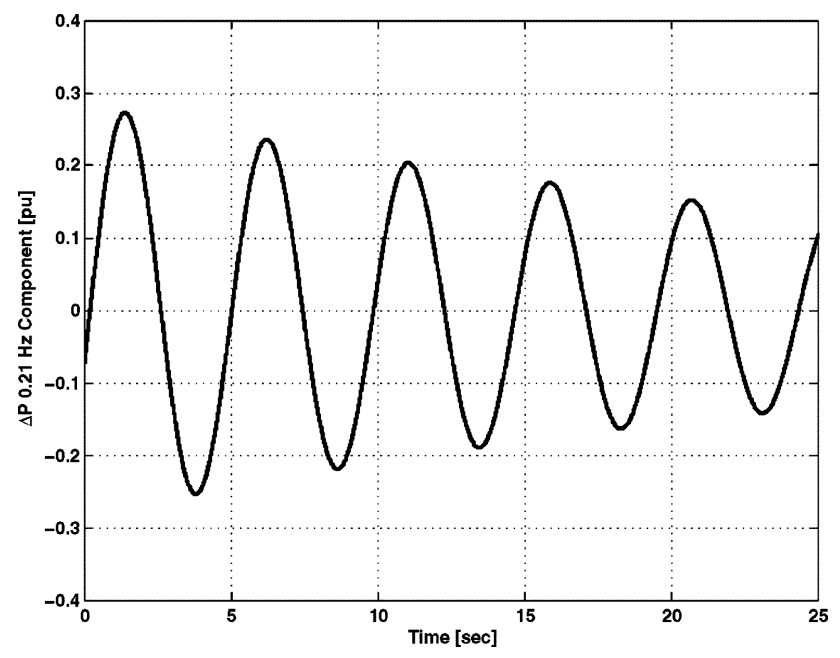

Fig. 7. $0.21 \mathrm{~Hz}(1.31 \mathrm{rad} / \mathrm{s})$ modal component.

two pairs of lightly damped modes and a single real mode. The lightly damped modes are the inter-area mode (Modes 9 and 10 in Table I) and a second lightly damped mode that results from the self combination of $\lambda_{9}$ and $\lambda_{10}: \lambda_{9}+\lambda_{9}\left(\lambda_{9,9}=\right.$ $-0.116+\mathrm{j} 2.62)$ and $\lambda_{10}+\lambda_{10}\left(\lambda_{10,10}=-0.116-\mathrm{j} 2.62\right)$.

Figs. 7 and 8 show the two modal components that constitute the $\Delta \mathrm{P}$. In other words, $\Delta \mathrm{P}$ in Fig. 6 , is equal to the sum of the signals shown in Figs. 7 and 8 (plus an offset given by the real mode).

It is not the purpose of this discussion to delve into system identification issues, but rather to point out the possibility of detecting higher modes in the transient response of a system. The presence of this type of modes is of particular significance in cases for which the linearized form of the system is not available. It is thus pertinent to inquire if the possibility exists of extending the understanding of a system dynamic performance beyond the linear region by applying alternative analysis methods such as Normal Form analysis.

\section{B. Normal Forms Analysis}

Normal Forms analysis is based on the system representation given by (13). Of particular interest are the second-order modes,

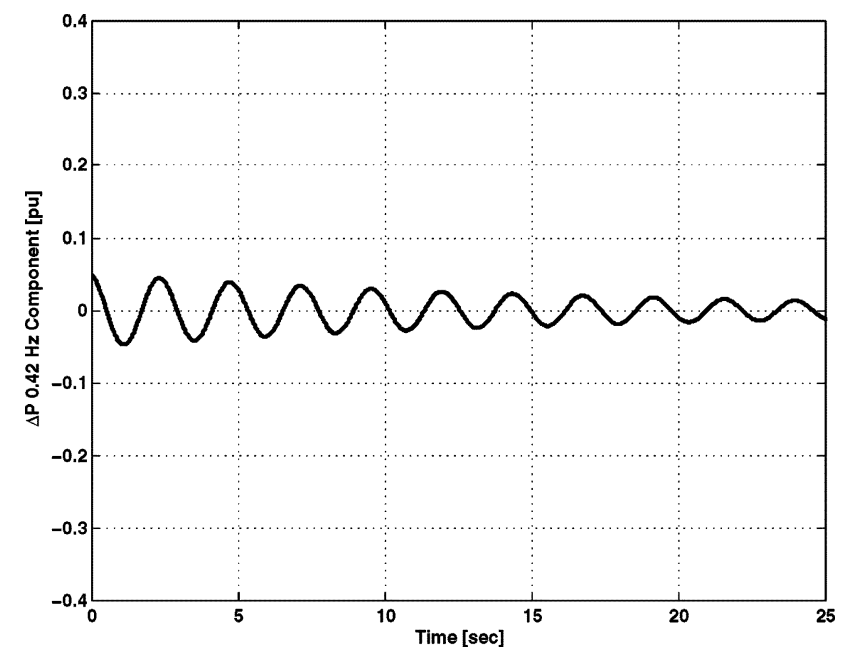

Fig. 8. $0.42 \mathrm{~Hz}(2.62 \mathrm{rad} / \mathrm{s})$ modal component.
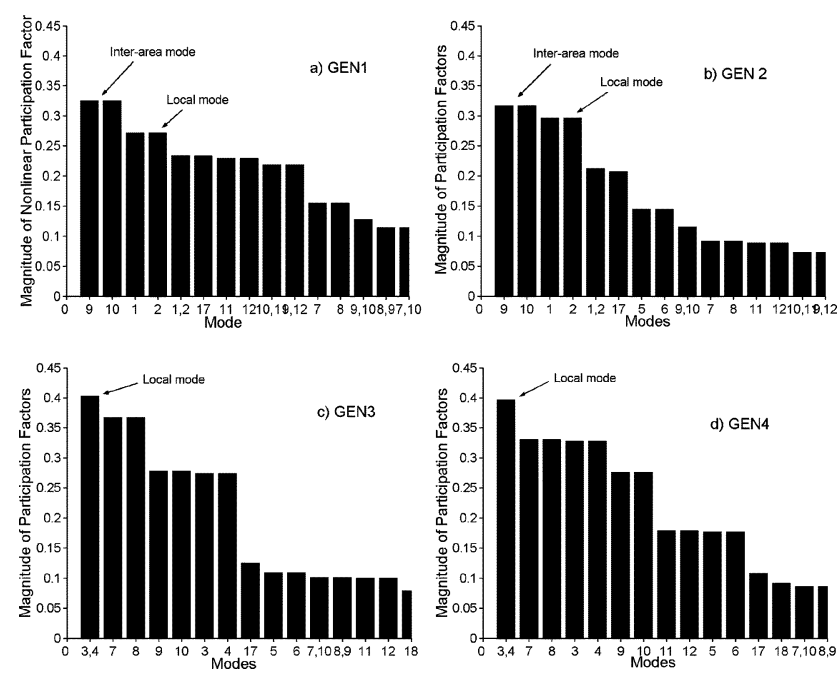

Fig. 9. Approximate nonlinear participation factors.

$\lambda_{k}+\lambda_{l}$, that contribute to the system response. The significance of these modes can be determined using the nonlinear participation factors and nonlinear interaction indices. Specifically, by exciting a given mode of concern, the rotor speed deviation of the system generators can be expressed in the form

$$
\Delta \omega_{i}(t)=\sum_{j=1}^{n} p 2_{i j} e^{\lambda_{j} t}+\sum_{k=1}^{n} \sum_{l=k}^{n} p 2_{k l}^{i} e^{\left(\lambda_{k}+\lambda_{l}\right) t}, i=1, \ldots, 4
$$

where $p 2_{i j}$ and $p 2_{k l}^{i}$ are the second-order nonlinear participation factors defined in (20). The nonlinear mode-state participation factors for the speed states are shown in Fig. 9.

Fig. 9 shows the largest 15 nonlinear participation factors $\left(p 2_{i j}, p 2_{k l}^{i}\right)$ for the four generators as a function of the interacting modes. The nonlinear participation factors for machines in Area 1 indicate the dominant presence of the inter-area Mode 9. This is similar to results obtained using linear analysis. On the other hand, and in contrast to the results obtained using the linear participation factors, the nonlinear participation factors for the machines in Area 2 indicate that the combination Mode $(3,4)\left(\lambda_{3,4}=-3.604\right)$, and not Mode 9, is the predominant mode. The combination Mode $(3,4)$ is followed by Mode 7 and, 
to a lesser extent, by the inter-area Mode 9. Furthermore, the participation of Modes 7, 8, 11, 12, and the combination modes $(7,10)$ and $(8,9)$ in Fig. 9(c) and (d), in the speed deviations of machines GEN3 and GEN4 suggest the potential for nonlinear modal interactions. It should be noted that the amplitude of the combination mode $(3,4)$ decays rapidly compared with Mode 9.

The Nonlinear Interaction Index, $I I(j)$, introduced in Section IV provides additional information on the modal interactions. The values of $I I(j)$ computed based on a $34 \mathrm{~ms}$ fault at Bus 6 reveal a strong modal interaction between the inter-area Mode 9, and Modes 11, 12 along with Modes 5 through 7. In addition, Modes 3, 5, and 7 strongly interact with Mode 11. An interesting aspect of these results is that at the instant of fault clearing the self interaction of Mode $9\left(\lambda_{9}+\lambda_{9}\right)$ is much smaller than the interaction of Mode 9 with other modes [29].

\section{Discussion}

The results presented in the preceding paragraphs illustrate differences and similarities between the linear and nonlinear analyzes. Both approaches describe the natural response of the system as a sum of complex exponential terms. However, whereas the state variables computed using linear analysis consist of a sum of terms that only include individual modes, the state variables computed using Normal Forms include not only the individual modes, but also combination modes of the form $\lambda_{k}+\lambda_{l}$ that describe modal interactions not observable using linear analysis. Depending on the system operating conditions, these terms may represent system dynamic characteristics that are significantly different than those expected from a linear analysis. To illustrate this important fact, a further analysis of the nonlinear phenomena is provided in [29] by addressing three specific issues: 1 ) existence of a $0.1-\mathrm{Hz}$ component (approx.) in the system response not characterized by the $0.1-\mathrm{Hz}$ mode listed in Table I; 2) computation of modal interactions for the $0.1-\mathrm{Hz}$ component using initial conditions evaluated at significantly different times; 3 ) existence of the second-order harmonic of the inter-area mode. Here, only the first item is considered.

Mode 11 is a very well damped mode (see Table IX) and, from a linear analysis point of view, it is not expected to play a significant role in the system response once it decays as dictated by its damping. However, in the course of this investigation, the analysis of the system response using Intrinsic Mode Functions and the Hilbert transform [32] suggested the presence of a $0.1-\mathrm{Hz}$ component that persists beyond the time frame associated with the $0.1-\mathrm{Hz}$ mode listed in Table IX. These results led to a closer scrutiny of the system response as given by with emphasis on those modes that lead to a $0.1-\mathrm{Hz}$ component. More specifically, the following question was considered: Are there terms in the speed of GEN4, i.e.,

$\Delta \omega_{i}(t)=\sum_{j=1}^{n} u_{i j} z_{j 0} e^{\lambda_{j} t}+\sum_{j=1}^{n} u_{i j}\left[\sum_{k=1}^{n} \sum_{l=1}^{n} h 2_{k l}^{j} z_{k o} z_{l o} e^{\left(\lambda_{k}+\lambda_{l}\right) t}\right]$

that give rise to a $0.1-\mathrm{Hz}$ component that persists during the time frame $0<t<40 \mathrm{~s}$ ? The answer to this question is obtained by considering the combinations of second-order modes that result
TABLE $\mathrm{X}$

Modal Combinations That Result IN $\approx 0.1 \mathrm{~Hz}$

\begin{tabular}{|c|c|c|c|c|}
\hline $\begin{array}{c}\text { Mode \# } \\
\boldsymbol{k}\end{array}$ & $\begin{array}{c}\text { Mode \# } \\
\boldsymbol{l}\end{array}$ & Mode & Freq. (Hz) & $\begin{array}{c}\text { Damping } \\
\text { Ratio (\%) }\end{array}$ \\
\hline 9 & 14 & $-0.349+\mathrm{j} 0.83$ & 0.130 & 38.76 \\
\hline 10 & 13 & $-0.349-\mathrm{j} 0.83$ & 0.130 & 38.76 \\
\hline 9 & 16 & $-0.382+\mathrm{j} 0.83$ & 0.133 & 41.81 \\
\hline 10 & 15 & $-0.382-\mathrm{j} 0.83$ & 0.133 & 41.81 \\
\hline 5 & 5 & $-7.54+\mathrm{j} 0.76$ & 0.120 & 99.50 \\
\hline 6 & 6 & $-7.54-\mathrm{j} 0.76$ & 0.120 & 99.50 \\
\hline 9 & 12 & $-1.11+\mathrm{j} 0.66$ & 0.105 & 85.85 \\
\hline 10 & 11 & $-1.11-\mathrm{j} 0.66$ & 0.105 & 85.85 \\
\hline
\end{tabular}

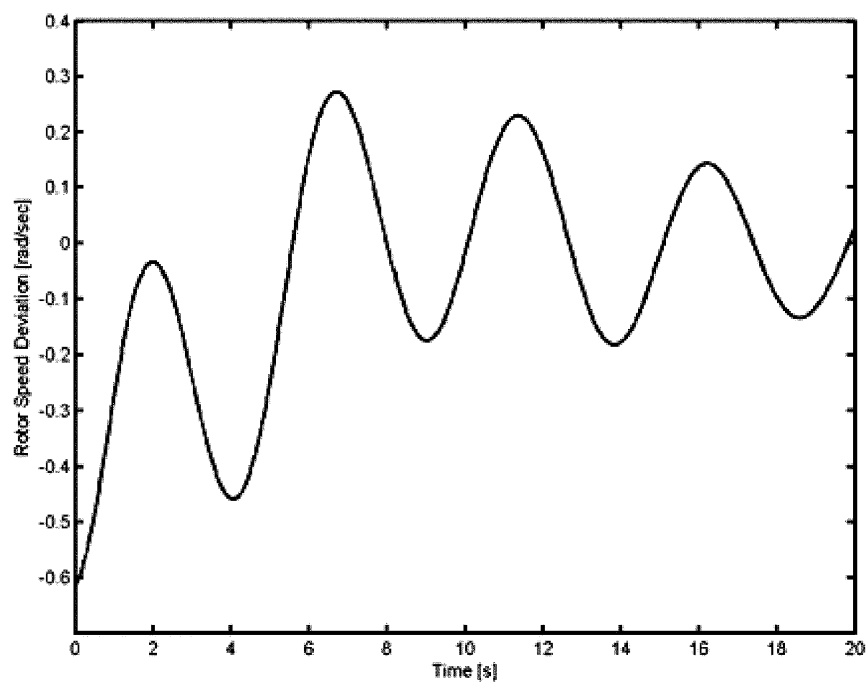

Fig. 10. Time response of mode $9,14,16,(9,14)$, and $(9,16)$.

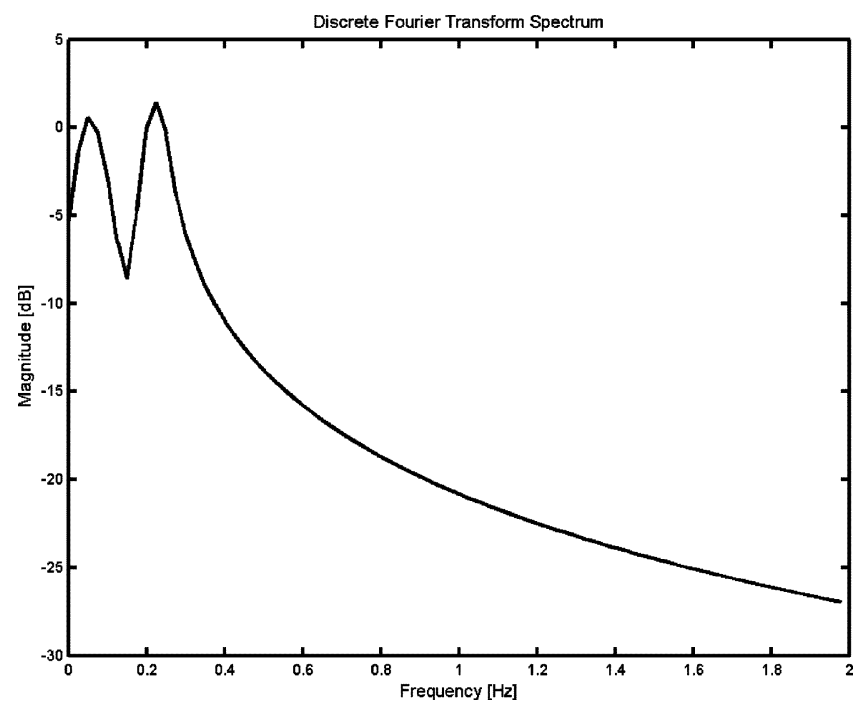

Fig. 11. DFT of time response in Fig. 7.

in a frequency of approximately $0.1 \mathrm{~Hz}$. These modes and their associated frequencies are shown in Table X.

The time response (A.2) computed using the terms in Table $\mathrm{X}$ and the terms corresponding to Mode 9 is shown in Fig. 10; its discrete Fourier transform (DFT) is shown in Fig. 11. The DFT clearly shows the presence of two dominant components at 0.1 and $0.2 \mathrm{~Hz}$. For comparison, Fig. 12 shows the time response (A.1) including only terms related to the inter-area mode, Mode 


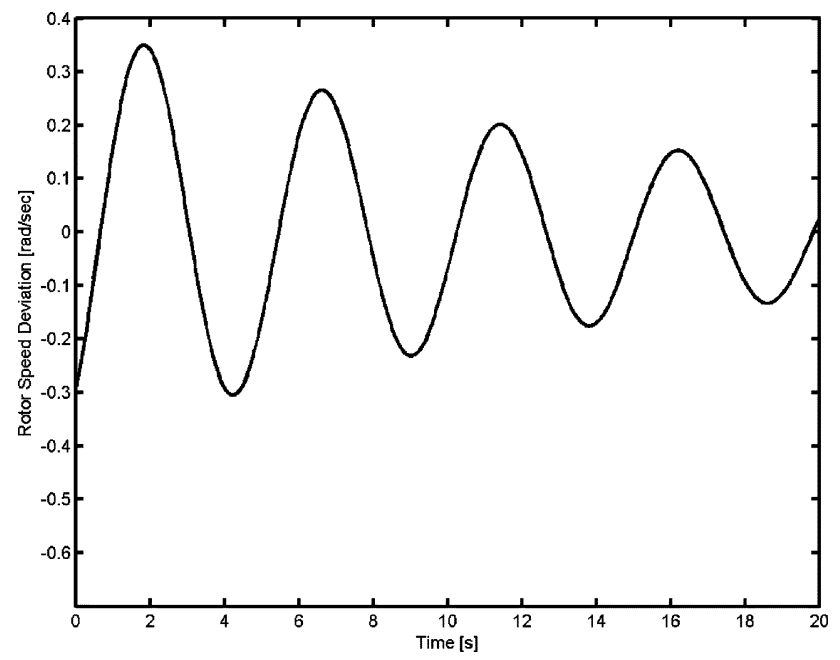

Fig. 12. Response of generator 4 including only terms corresponding to mode 9 .

9. The reason for the presence of the $0.1-\mathrm{Hz}$ component is provided by the combination modes in Table III: the first four entries correspond to two $0.1-\mathrm{Hz}$ modes with damping ratios of $39 \%$ and $42 \%$. These modes arise from the interaction of the inter-area mode and other modes and, although well damped, will be observable for a significant time span. This analysis reinforces the main claim of the Normal Form analysis that nonlinear interaction of modes may lead to frequency components not observed by linear modal identification techniques.

\section{APPENDIX B}

\section{CONTROller Placement Using Normal Forms}

Appendix B serves the purpose of suggesting possible avenues for further research by describing the possible use of Normal Forms for the siting of damping controllers. For a more extensive description, the interested reader is referred to [33]. It is emphasized that PSSs are employed for illustrative purposes only; other devices may be more appropriate for addressing systemic dynamic issues.

Specifically, the nonlinear participation factors are used to determine the best site for the PSS. By exciting a given mode of concern, the rotor speed deviation of the system generators is expressed in the form

$$
\Delta \omega_{i}(t)=\sum_{j=1}^{n} p 2_{i j} e^{\lambda_{j} t}+\sum_{k=1}^{n} \sum_{l=k}^{n} p 2_{k l}^{i} e^{\left(\lambda_{k}+\lambda_{l}\right) t}, i=1, \ldots, 4
$$

where $p 2_{i j}$ and $p 2_{k l}^{i}$ the second-order nonlinear participation factors have been defined in (19) and (20). For these states, the nonlinear mode-state participations were derived

Fig. 13 shows the top 15 participation factors for the machines as a function of the interacting modes obtained from (B.1). In evaluating these participation factors, the procedure described in Section II where only the excited state has an entry in the
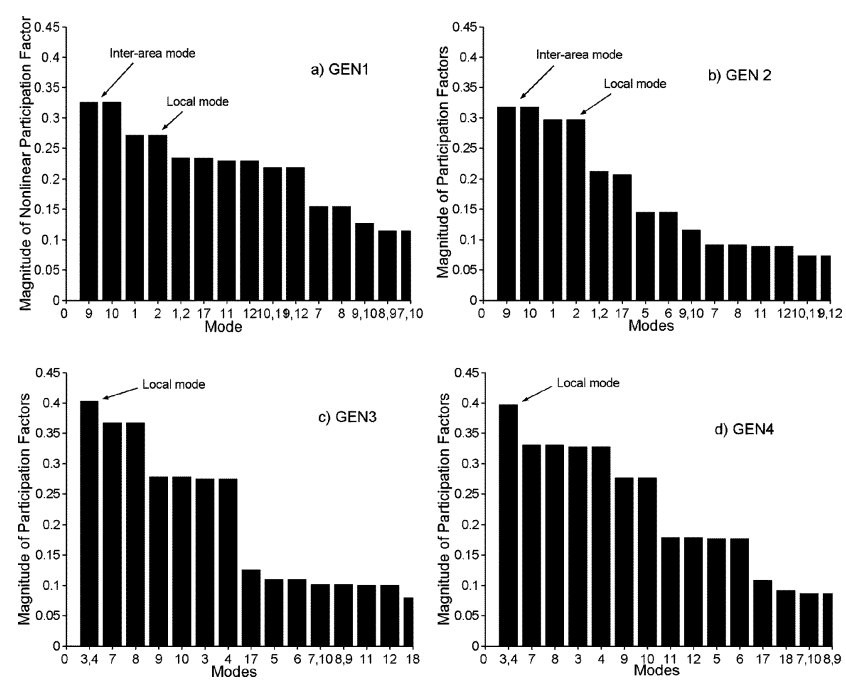

Fig. 13. Approximate nonlinear participation factors.

TABLE XI

COMPARISON OF NONLINEAR PARTICIPATION FACTORS

\begin{tabular}{l|c|c}
\hline Machine & $\begin{array}{c}\text { Analytical } \\
\text { Approximation/ Ranking }\end{array}$ & $\begin{array}{c}\text { Numerical Estimate/ } \\
\text { Ranking }\end{array}$ \\
\hline GEN1 & $\mathbf{0 . 3 2 5 7}(1)$ & $0.3170(2)$ \\
\hline GEN2 & $0.3174(2)$ & $\mathbf{0 . 3 4 0 7}(1)$ \\
\hline GEN3 & $0.2786(3)$ & $0.2845(3)$ \\
\hline GEN4 & $0.2765(4)$ & $0.2794(4)$ \\
\hline
\end{tabular}

unit vector $e_{j}$ was used. Further, numerical estimates for nonlinear participation factors derived using the numerically obtained initial condition $z_{0}$ for the specific scenario considered were then used to determine the best location for power system stabilizers. The nonlinear participation factors for machines in Area 1 shown in Fig. 13(a) and (b) show the dominant presence of the inter-area Mode 9, suggesting that Area 1 is the best place to install PSSs. By contrast, the nonlinear participation factors for the machines in Area 2 indicate the presence of the combination local Mode $(3,4)$ followed by the presence of Mode 7 , and, to a lesser extent, by the inter-area Mode 9 . In contrast to linear participation factors, nonlinear participation factors suggest that Area 1 is a better location for the PSSs. Further, the large participation of Modes 7, 8, and 11, 12 in the speed deviations of machines GEN3 and GEN4 suggests the potential for undesirable nonlinear modal interaction arising from this phenomenon [see Fig. 13(c) and (d)].

Table XI shows the nonlinear participation factors computed using the approximations and the exact $z_{0}$ for the scenario. The results single out machine GEN2 as the best location for siting the PSS.

\section{A. Second-Order Interaction Coefficients}

The analysis presented above is further reinforced using the second-order interaction coefficient analysis. To this end, a three-phase fault is applied at Bus 6 and is removed in $34 \mathrm{~ms}$ to investigate nonlinear effects arising from modal interaction. Normal Form analysis is conducted on the post-contingency condition. 
TABLE XII

NONLINEAR INTERACTION COEFFICIENTS FOR KEY MODES

\begin{tabular}{|c|c|c|c|}
\hline $\begin{array}{c}\text { Mode } \\
\boldsymbol{i}\end{array}$ & $\mid h_{2_{k l}} z_{k o} z_{l o}$ & $\boldsymbol{j}$ & $\boldsymbol{k}$ \\
\hline 1,2 & 8.04953 & 1 & 2 \\
\hline 3,4 & 12.89761 & 11 & 12 \\
\hline 5,6 & 9.83190 & 11 & 12 \\
\hline 7,8 & 18.24392 & 11 & 11 \\
\hline \multirow{5}{*}{9} & 94.40 & $\mathbf{1 1}$ & $\mathbf{1 2}$ \\
& 82.03651 & $\mathbf{1 1}$ & $\mathbf{1 1}$ \\
& 49.05541 & 12 & 12 \\
& 24.91597 & 7 & 11 \\
& 24.77925 & 7 & 12 \\
& 22.76055 & 6 & 12 \\
& 21.90442 & 5 & 11 \\
& 17.53426 & 11 & 13 \\
& 16.77551 & 8 & 11 \\
& 15.26967 & 6 & 11 \\
& 13.64500 & 5 & 12 \\
& 12.81368 & 11 & 14 \\
& 12.31203 & 8 & 12 \\
\hline
\end{tabular}

TABLE XIII

EFFECT OF PSSS ON SYSTEM DAMPING

\begin{tabular}{|c|c|c|c|}
\hline $\begin{array}{c}\text { PSS } \\
\text { location }\end{array}$ & Eigenvalues & $\begin{array}{c}\text { Frequency } \\
\text { (Hz) }\end{array}$ & $\begin{array}{c}\text { Damping } \\
\text { ratio (\%) }\end{array}$ \\
\hline GEN1 & $-0.1151 \pm \mathrm{j} 1.38(9)$ & 0.220 & 8.30 \\
& $-1.355 \pm \mathrm{j} 7.71(1)$ & 1.227 & 17.30 \\
\hline GEN2 & $\mathbf{- 0 . 1 5 3 4} \pm \mathbf{j} 1.43(\mathbf{9})$ & 0.223 & 10.87 \\
& $-1.303 \pm \mathrm{j} 7.73(1)$ & 1.231 & 16.61 \\
\hline GEN3 & $-0.0354 \pm \mathrm{j} 1.30(9)$ & 0.206 & 2.81 \\
& $-1.940 \pm \mathrm{j} 7.54(3)$ & 1.199 & 24.93 \\
\hline GEN4 & $-0.0371 \pm \mathrm{j} 1.28(9)$ & 0.203 & 2.90 \\
& $-1.846 \pm \mathrm{j} 7.54(3)$ & 1.200 & 23.78 \\
\hline
\end{tabular}

The analysis of nonlinear interaction coefficients in Table XII reveals strong modal interaction between the inter-area mode and the control Modes 5 and 7, in addition to Mode 11. In turn, Modes 3, 5, and 7 are seen to strongly interact with Mode 11. The interaction coefficients also show that the self interaction of Mode 9 is much smaller than the interaction of Mode 9 with other modes, and that self interaction of Mode 11 produces a mode with the same frequency characteristics as the inter-area mode but different in damping.

\section{B. Small-Signal Control Design}

To verify the effects of nonlinear behavior on the analysis and design of controllers, PSSs were designed using the approach in [34], [35]. In this analysis, a PSS was designed considering a single machine location at a time. Table XIII gives the local mode eigenvalues for each area and the inter-area mode along with the damping ratio and frequency of oscillation for each control alternative. Small-signal results show that the use of PSSs in machines in Area 1 results in more damped behavior. Thus, for instance, for the case with a PSS at machine GEN2, the damping ratio is increased to $10.87 \%$. By contrast, adding PSSs at machines in Area 2 may actually decrease the damping of the inter-area mode from $4.4 \%$ to $2.81 \%$ and $2.90 \%$ for the cases with a PSS added to machines GEN1 and GEN2, respectively. Comparing these results with the analysis of nonlinear partici- pation factors in Fig. 13, one can see that modal interaction is not properly captured by linear analysis. As a result, conventional techniques do not necessarily identify the ideal location for damping controllers.

\section{Discussion}

The primary goal of the analysis presented in this section was to address question 3 in the task force objectives with a concrete example. The case described in this section clearly demonstrates that for a highly stressed case, conventional linear techniques to locate controllers could provide results that are not necessarily ideal to damp oscillations. The engineering insight provided by the nonlinear participation factors and the interactions analysis clearly indicates that the location of the controllers in Area 1 is better suited to damp the oscillations because of the high degree of interaction that takes place. The design of the PSS and the small-signal testing using the setting obtained demonstrate the efficacy of the new locations and their ability to effectively improve the damping of the inter area mode. The analysis also reinforces the importance of nonlinear modal interaction and demonstrates: 1) the ability of the method of Normal Forms to correctly identify the interaction and 2) the efficacy of the various indices to accurately quantify the level of interaction and predict the states that are involved in it.

\section{APPENDIX C SYSTEM DATA}

\section{A. Data for Case Study in Section IV}

Tie Line Flow $400 \mathrm{MW}$ :

TABLE XIV

LINE DATA-IN PU ON A 100 MVA BASE

\begin{tabular}{|c|c|c|c|}
\hline From Bus & To Bus & R & X \\
\hline 1 & 2 & 0.0025 & 0.0250 \\
\hline 2 & 5 & 0.0010 & 0.0100 \\
\hline 5 & 6 & 0.0220 & 0.2200 \\
\hline 3 & 4 & 0.0025 & 0.0250 \\
\hline 4 & 6 & 0.0010 & 0.0100 \\
\hline
\end{tabular}

TABLE XV

LOAD DATA

\begin{tabular}{|c|c|c|}
\hline Bus \#i & Load (MW) & Load (MVAR) \\
\hline 5 & 900 & 250 \\
\hline 6 & 1400 & 250 \\
\hline
\end{tabular}

TABLE XVI

Generator Damping In PU ON MAchine Base

\begin{tabular}{|c|c|}
\hline Generator & D \\
\hline 1 & 9.0 \\
\hline 2 & 1.0 \\
\hline 3 & 11.0 \\
\hline 4 & 1.2 \\
\hline
\end{tabular}


TABLE XVII

GenERATOR DATA on MACHINE BASE (G1,G2,G3,G4)

\begin{tabular}{|c|c|c|c|}
\hline Parameter & Value & Parameter & Value \\
\hline$R_{a}$ & 0.0025 & $x_{q}^{\prime \prime}$ & 0.0 \\
\hline$x_{d}$ & 1.80 & $\tau_{d 0}^{\prime}$ & 8.0 \\
\hline$x_{q}$ & 1.70 & $\tau_{q 0}^{\prime}$ & 0.40 \\
\hline$x_{\ell}$ & 0.0 & $\tau_{d 0}^{\prime \prime}$ & 0.0 \\
\hline$x_{d}^{\prime}$ & 0.30 & $\tau_{q 0}^{\prime \prime}$ & 0.0 \\
\hline$x_{q}^{\prime}$ & 0.30 & $\mathrm{MVA}_{\text {base }}$ & 900 \\
\hline$x_{d}^{\prime \prime}$ & 0.0 & $\mathrm{H}$ & 6.5 \\
\hline
\end{tabular}

TABLE XVIII

EXCITER DATA (IEEE TYPES ST1, AC4 AND AC4A)

\begin{tabular}{|c|c|c|c|c|c|c|}
\hline $\mathbf{K}_{\mathbf{A}}$ & $\mathbf{T}_{\mathbf{A}}$ & $\mathbf{T}_{\mathbf{C}}$ & $\mathbf{T}_{\mathbf{B}}$ & $\mathbf{T}_{\mathbf{R}}$ & $\mathbf{V}_{\mathbf{R M A X}}$ & $\mathbf{V}_{\mathbf{R M I N}}$ \\
\hline 100 & 0.01 & 1.0 & 10.0 & 0.01 & 5.0 & -5.0 \\
\hline
\end{tabular}

TABLE XIX

Shunt Susceptance B (P.U. ON 100 MVA BASE)

\begin{tabular}{|c|c|}
\hline Bus \# & B \\
\hline 5 & 2.5510 \\
\hline 6 & 2.5430 \\
\hline
\end{tabular}

\section{B. Data for Case Study in Appendix A}

Tie Line Flow $410 \mathrm{MW}$ :

TABLE XX

LOAD DATA

\begin{tabular}{|c|c|c|}
\hline Bus \# & Load (MW) & Load (MVAR) \\
\hline 5 & 890 & 250 \\
\hline 6 & 1410 & 250 \\
\hline
\end{tabular}

TABLE XXI

GENERATOR DAMPING IN PU ON MACHINE BASE

\begin{tabular}{|c|c|}
\hline Generator & D \\
\hline 1 & 4.0 \\
\hline 2 & 2.0 \\
\hline 3 & 11.0 \\
\hline 4 & 10.0 \\
\hline
\end{tabular}

TABLE XXII

EXCITER GAIN

\begin{tabular}{|c|c|}
\hline Generator & $\mathbf{K}_{\boldsymbol{\Lambda}}$ \\
\hline 1 & 180 \\
\hline 2 & 100 \\
\hline 3 & 130 \\
\hline 4 & 220 \\
\hline
\end{tabular}

\section{REFERENCES}

[1] "Inter-Area Oscillations in Power Systems," IEEE Publication Catalog Number 95 TP 101, 1995.

[2] V. Vittal, N. Bhatia, and A. A. Fouad, "Analysis of the inter-area mode phenomenon in power systems following large disturbances," IEEE Trans. Power Syst., vol. 6, no. 4, pp. 1515-1521, Nov. 1991.

[3] C.-M. Lin, V. Vittal, W. Kliemann, and A. A. Fouad, "Investigation of modal interaction and its effects on control performance in stressed power systems using normal forms of vector fields," IEEE Trans. Power Syst., vol. 11, no. 2, pp. 781-787, May 1996.

[4] Y. X. Ni, V. Vittal, W. Kliemann, and A. A. Fouad, "Nonlinear modal interaction in HVDC/AC power systems with D. C. modulation," IEEE Trans. Power Syst., vol. 11, no. 4, pp. 2011-2017, Nov. 1996.

[5] J. Thapar, V. Vittal, W. Kliemann, and A. A. Fouad, "Application of the normal form of vector fields to predict interarea separation in power systems," IEEE Trans. Power Syst., vol. 12, no. 2, pp. 844-850, May 1997.

[6] S. Saha, A. A. Fouad, W. Kliemann, and V. Vittal, "Stability boundary approximation of a power system using the real normal form of vector fields," IEEE Trans. Power Syst., vol. 12, no. 2, pp. 797-802, May 1997.

[7] Y. Ni, V. Vittal, and W. Kliemann, "A study of system separation mechanisms in the neighborhood of a relevant type-n UEP via normal form of vector fields," Proc. Inst. Elect. Eng., Gener., Transm., Distrib., vol. 145, no. 2, pp. 139-144, Mar. 1998.

[8] S. K. Starrett and A. A. Fouad, "Nonlinear measures of mode-machine participation,” IEEE Trans. Power Syst., vol. 13, no. 2, pp. 389-394, May 1998.

[9] G. Jang, V. Vittal, and W. Kliemann, "Effect of nonlinear modal interaction on control performance: Use of normal forms technique in control design, Part 1: General theory and procedure," IEEE Trans. Power Syst., vol. 13, no. 2, pp. 401-413, May 1998.

[10] - "Effect of nonlinear modal interaction on control performance: Use of normal forms technique in control design, Part II: Case studies," IEEE Trans. Power Syst., vol. 13, no. 2, pp. 414-421, May 1998.

[11] V. Vittal, W. Kliemann, Y.-X. Ni, D. G. Chapman, A. D. Silk, and D. J. Sobajic, "Determination of generator groupings for an islanding scheme in the Manitoba Hydro system using the method of normal forms," IEEE Trans. Power Syst., vol. 13, no. 4, pp. 1345-1351, Nov. 1998.

[12] S. Zhu, V. Vittal, and W. Kliemann, "Analyzing dynamic performance of power systems over parameter space using normal forms of vector fields Part I: Identification of vulnerable regions," IEEE Trans. Power Syst., vol. 16, no. 3, pp. 444-450, Aug. 2001.

[13] - "Analyzing dynamic performance of power systems over parameter space using normal forms of vector fields Part II: Comparison of system structure," IEEE Trans. Power Syst., vol. 16, no. 3, pp. 451-455, Aug. 2001.

[14] M. Klein, G. J. Rogers, and P. Kundur, "A fundamental study of interarea oscillations in power systems," IEEE Trans. Power Syst., vol. 6, no. 3, pp. 914-921, Aug. 1991.

[15] D. K. Arrowsmith and C. M. Place, An Introduction to Dynamical Systems. Cambridge, U.K.: Cambridge Univ. Press, 1990.

[16] V. I. Arnold, Geometrical Methods in the Theory of Ordinary Differential Equations. New York: Springer-Verlag, 1983.

[17] J. Guckenheimer and P. Holmes, Nonlinear Oscillations, Dynamical Systems, and Bifurcations of Vector Fields. New York: Springer-Verlag, 1983.

[18] S. Wiggins, Introduction to Applied Nonlinear Dynamical Systems and Chaos. New York: Springer-Verlag, 1990.

[19] A. H. Nayfeh, Method of Normal Forms. New York: Wiley, 1993.

[20] S. K. Starrett, V. Vittal, A. A. Fouad, and W. Kliemann, "A methodology for the analysis of nonlinear, interarea interactions between power system natural modes of oscillation utilizing normal forms," in Proc. 1993 Int. Symp. Nonlinear Theory Application, vol. 2, Waikiki, HI, Dec. 5-10, 1993, pp. 523-538.

[21] S. K. Starrett, W. Kliemann, V. Vittal, and A. A. Fouad, "Power system modal behavior: Significance of second and third order nonlinear terms," in Proc. North American Power Symp., Washington, DC, Oct. 1993, pp. 241-255.

[22] I. J. Perez-Arriaga, G. C. Verghese, and F. C. Schweppe, "Selective modal analysis with applications to electric power systems. Part 1: Heuristic introduction," IEEE Trans. Power App. Syst., vol. PAS-101, pp. 3117-3125, Sep. 1982. 
[23] S. K. Starrett, "Application of normal forms of vector fields to stressed power systems," Ph.D. dissertation, Iowa State Univ., Ames, IA, 1994.

[24] I. Dobson and E. Barocio, "Scaling of normal form analysis coefficients under coordinate change," IEEE Trans. Power Syst., vol. 19, no. 3, pp. 1438-1444, Aug. 2004.

[25] N. Kshatriya, U. D. Annakkage, A. M. Gole, and I. T. Fernando, "Improving the accuracy of normal form analysis," IEEE Trans. Power Syst., vol. 20, no. 1, pp. 286-293, Feb. 2005.

[26] E. Barocio and A. R. Messina, "Application of perturbation methods to analysis of low frequency inter-area oscillations," in Proc. 2000 IEEE Power Engineering Society Summer Meeting, Seattle, WA.

[27] J. F. Hauer, C. J. Demeure, and L. L. Scharf, "Initial results in Prony analysis of power system response signals," IEEE Trans. Power Syst., vol. 5, no. 1, pp. 80-89, Feb. 1990.

[28] A. R. Messina and V. Vittal, "Assessment of nonlinear interaction between nonlinearly coupled modes using higher-order spectra," IEEE Trans. Power Syst., vol. 20, no. 1, pp. 375-383, Feb. 2005.

[29] J. J. Sanchez-Gasca, V. Vittal, M. J. Gibbard, A. R. Messina, D. J. Vowles, S. Liu, and U. D. Annakkage, "Analysis of higher order terms for small signal stability analysis," in Proc. Power Engineering Society General Meeting 2005, San Francisco, CA, Jun. 2005, ID: 05GM411.
[30] SSAT: Small Signal Analysis Tool, Powertech Labs Inc., Canada. [Online]. Available: http://www.powertechlabs.com.

[31] J. Juang and R. S. Pappa, "An eigensystem realization algorithm for modal parameter identification and model reduction," J. Guidance Control, vol. 8, no. 5, pp. 620-627, Sep.-Oct. 1985.

[32] N. E. Huang, Z. Shen, S. R. Long, M. C. Wu, H. H. Shih, Q. Zheng, N. Yen, C. C. Tung, and H. H. Liu, "The empirical mode decomposition and the hilbert spectrum for nonlinear and nonstationary time series analysis," in Proc. R. Soc. London A, vol. 454, 1998, pp. 903-995.

[33] S. Liu, A. R. Messina, and V. Vittal, "Assessing placement of controllers and nonlinear behavior using normal form analysis," IEEE Trans. Power Syst., vol. 20, no. 3, pp. 1486-1495, Aug. 2005.

[34] P. Kundur, M. Klein, G. J. Rogers, and M. S. Zywno, "Application of power system stabilizers for enhancement of overall system stability," IEEE Trans. Power Syst., vol. 4, no. 2, pp. 614-626, May 1989.

[35] E. V. Larsen and D. A. Swann, "Applying power system stabilizers Parts I, II, and III," IEEE Trans. Power App. Syst., vol. PAS-100, pp. 3017-3046, Jun. 1981. 